\title{
A Precision Measurement of pp Elastic Scattering Cross Sections at Intermediate Energies
}

\author{
D. Albers ${ }^{2}$, F. Bauer ${ }^{2}$, J. Bisplinghoff ${ }^{1}$, R. Bollmann ${ }^{2}$, K. Büßer ${ }^{2}$, M. Busch ${ }^{1}$, R. Daniel ${ }^{1}$, O. Diehl ${ }^{1}$, F. Dohrmann ${ }^{2}$, \\ H.P. Engelhardt ${ }^{1}$, J. Ernst ${ }^{1}$, P.D. Eversheim ${ }^{1}$, M. Gasthuber ${ }^{2}$, R. Gebel ${ }^{3}$, J. Greiff ${ }^{2}$, A. Groß ${ }^{2}$, R. Groß-Hardt ${ }^{1}$, S. Heider $^{1}$, \\ A. Heine ${ }^{1}$, F. Hinterberger ${ }^{1}$, T. Hüskes ${ }^{1}$, M. Igelbrink ${ }^{2}$, M. Jeske ${ }^{1}$, R. Langkau ${ }^{2}$, J. Lindlein $^{2}$, R. Maier $^{3}$, R. Maschuw $^{1}$, \\ F. Mosel $^{1}$, D. Prasuhn ${ }^{3}$, H. Rohdjeß ${ }^{1}$, D. Rosendaal ${ }^{1}$, P. von Rossen ${ }^{3}$, N. Scheid ${ }^{1}$, N. Schirm ${ }^{2}$, M. Schulz-Rojahn ${ }^{1}$, \\ F. Schwandt ${ }^{1}$, V. Schwarz ${ }^{1}$, W. Scobel ${ }^{2}$, S. Thomas ${ }^{1}$, H.-J. Trelle ${ }^{1}$, E. Weise ${ }^{1}$, A. Wellinghausen ${ }^{2}$, K. Woller ${ }^{2}$, and R. Ziegler ${ }^{1}$ \\ 1 Helmholtz-Institut für Strahlen- und Kernphysik, Universität Bonn, D-53115 Bonn, Germany \\ 2 Institut für Experimentalphysik, Universität Hamburg, D-22761 Hamburg, Germany \\ 3 Institut für Kernphysik, Forschungszentrum Jülich, D-52425 Jülich, Germany
}

December 17, 2013

\begin{abstract}
We have measured differential cross sections for pp elastic scattering with internal fiber targets in the recirculating beam of the proton synchrotron COSY. Measurements were made continuously during acceleration for projectile kinetic energies between 0.23 and $2.59 \mathrm{GeV}$ in the angular range $30^{\circ} \leq \theta_{\text {c.m. }} \leq 90^{\circ}$. Details of the apparatus and the data analysis are given and the resulting excitation functions and angular distributions presented. The precision of each data point is typically better than $4 \%$, and a relative normalization uncertainty of only $2.5 \%$ within an excitation function has been reached. The impact on phase shift analysis as well as upper bounds on possible resonant contributions in lower partial waves are discussed.
\end{abstract}

PACS. 25.40.Cm Elastic proton scattering - 13.75.Cs Nucleon-nucleon interactions - 13.85.Dz Elastic scattering 21.30.-x Nuclear forces

\section{Introduction}

Elastic nucleon-nucleon scattering is a process fundamental to understanding nuclear forces. Its precise experimental knowledge bears on such basic questions as the confinement of quarks, the limits to the validity of meson exchange models in the nuclear regime and a basis upon which to test QCD inspired models at intermediate energies. Its knowledge also forms the basis of a broad range of applications in nuclear and heavy ion physics, e.g. as ingredients to models of reaction dynamics, excited nuclear matter and transport phenomena. Consequently, many experimental and theoretical studies (see [1] and references therein) have been devoted to this subject. The data base has about doubled over the past decade [2, 3, 4, 5], and global phase shifts - a convenient tool of parameterizing existing experimental knowledge - are on a sound basis up to about $1 \mathrm{GeV}$ in kinetic beam energy [6, 7, 8].

Closer inspection shows, however, that the vast majority of the data is below $0.8 \mathrm{GeV}$ of kinetic beam energy. Above that beam energy, the data base used to be increasingly sparse and somewhat uncertain in normalization, hampering both reliable phase shift analysis and conclusions towards the physics of strong interaction.

Send offprint requests to: $\mathrm{H}$. Rohdjeß, HISKP, Universität Bonn, Nussallee 14-16, D-53115 Bonn, Germany

Correspondence to: rohdjess@iskp.uni-bonn.de
The EDDA experiment was designed to provide pp elastic scattering data up to $2.5 \mathrm{GeV}$ - precise and consistent in normalization - for all of the above purposes. In particular, it was meant to supply data for phase shift analysis up to that projectile energy, and to test claims [9] and predictions, e.g. [10, 11, 12, 13], of dibaryonic resonances. To this end EDDA measured spin-averaged differential cross sections [14] as well as transverse analyzing powers [15] and spin correlation coefficients [16], utilizing the proton beams available at the cooler synchrotron COSY [17] at FZ Jülich.

An account of the results has already been given in [14], based on roughly $40 \%$ of the data available now. The purpose of this paper is to describe experimental details and to present a more elaborate analysis. In particular, corrections for radiation damage of the target was not included in the analysis of Ref. [14] which lead to cross sections systematically smaller by $\approx 3 \%$ for momenta above about $2 \mathrm{GeV} / \mathrm{c}$. In addition, a refined separation of proton-carbon scattering events was accomplished by pattern recognition techniques, resulting in reduced statistical errors. In total the statistical precision was improved by up to a factor of 3 .

The experimental setup is described in Sect. 2 while Sect. 3 focuses on a detailed description of the data analysis including event selection, vertex and angle reconstruction, background subtraction and normalization. Sect. 4 presents angular distri- 
butions and excitation functions, Sect. 5] discusses their impact on phase shift analysis and hypothetical dibaryonic resonances.

\section{The EDDA experiment}

EDDA was conceived as an experiment using the internal beam of the cooler synchrotron COSY [17], so as to measure continuous excitation functions during beam acceleration. It is placed at a beam waist in one of the straight sections of COSY's racetrack shaped lattice. With the protons recirculating with about 1.0-1.5 MHz, inherently thin, polarized atomic beam targets for the measurement of spin observables can be utilized with sufficient luminosity. However, for cross section measurements as reported here, thin $\mathrm{CH}_{2}$-fiber targets have been used, as they allow for a continuous monitoring of the instantaneous luminosity.

\subsection{Targets and Detector}

$\mathrm{CH}_{2}$-fibers were strung horizontally between the prongs of a fork, which could be moved vertically by a magnet-driven linear actuator to put the fiber into (and out of) the COSY beam. The thickness of the fibers was chosen as a compromise between long beam-lifetimes and sufficient sturdiness when exposed to the beam. A cross-section of $4 \times 5 \mu \mathrm{m}^{2} \mathrm{CH}_{2}$ proved to be a good choice, allowing a few $10^{8}$ protons recirculating in the COSY ring at about $1.5 \mathrm{MHz}$ without target-failure. Target fibers were prepared using a microtome to cut $4 \mu \mathrm{m}$ fibers from a commercially available polypropylene $\left(\mathrm{CH}_{2}\right)$ foil of $5 \mu \mathrm{m}$ thickness. To prevent build-up of charges due to ionization by the COSY-beam, the surface was made conducting by a thin $\left(20 \mu \mathrm{g} / \mathrm{cm}^{2}\right)$ aluminum coating, such that the target was grounded through the supporting fork made from aluminum.

The background resulting from the carbon content (and the $\mathrm{Al}$ coating) needs to be subtracted offline; It was measured using $5 \mu \mathrm{m}$ thick carbon fibers suitably coated with $\mathrm{Al}$. $\mathrm{CH}_{2}-$ targets lose slowly hydrogen content upon beam exposure. Therefore, a reservoir of targets was kept inside the COSY vacuum to ensure essentially uninterrupted operation of EDDA during data taking. In first order, the relative normalization of the excitation functions is not affected by the slow hydrogen loss since the time period of about $2 \mathrm{~s}$ for measuring a complete excitation function during beam acceleration is small with respect to the hydrogen loss rate.

The detector as used for cross section measurements is shown schematically in Fig. 1 A scintillator hodoscope surrounds the beam pipe downstream the target covering the angular range $10^{\circ} \leq \theta_{\text {lab }} \leq 72^{\circ}$ and subtending about $85 \%$ of $4 \pi$ in the c.m. for proton-proton elastic scattering. It consists of scintillator bars and rings designed to measure the points, at which the scattered proton and its recoil partner traverse the hodoscope in terms of azimuthal and polar angle $\left(\varphi_{1,2}\right.$ and $\theta_{\text {lab }, 1,2}$, respectively). The kinematic relationships between these angles (see Sect. 3.6) are used to identify elastic scattering events.

The inner hodoscope layer consists of 32 scintillator bars made of $\mathrm{BC} 408$ and read out on both ends via lucite light guides with Hamamatsu R1355 photomultipliers (PM) [18]. The scintillator bars have a triangular cross section (Fig. 2 (a)). Their

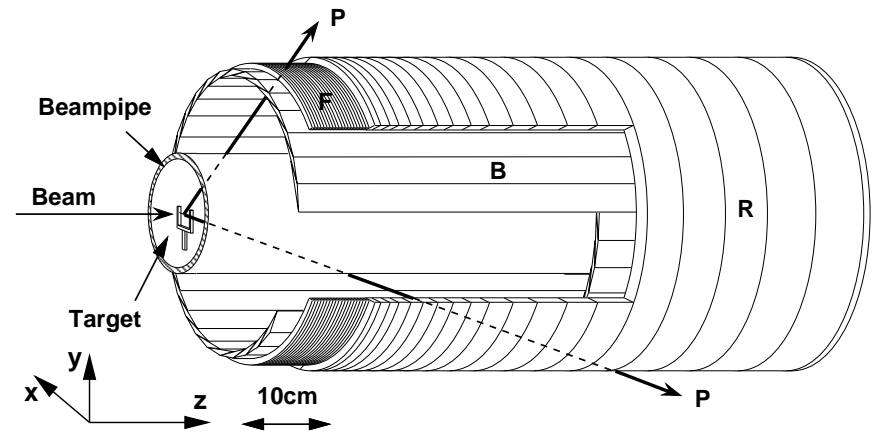

Fig. 1. The EDDA detector (not to scale): target: fiber $\left(\mathrm{CH}_{2}\right.$ or $\left.\mathrm{C}\right)$; B: scintillator bars; R: scintillator semi-rings; F: semi-rings made of scintillating fibers.

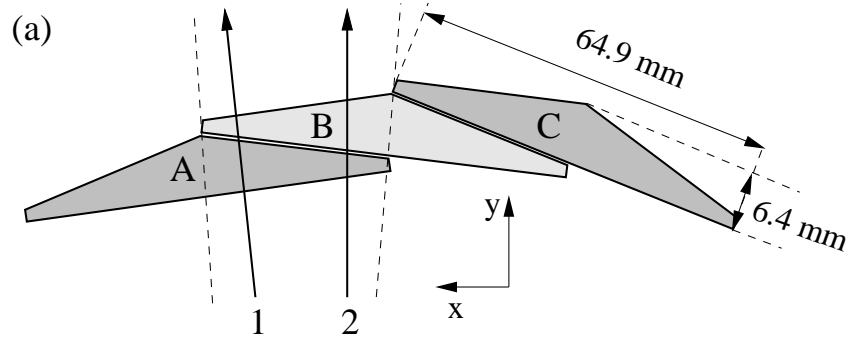

(b)

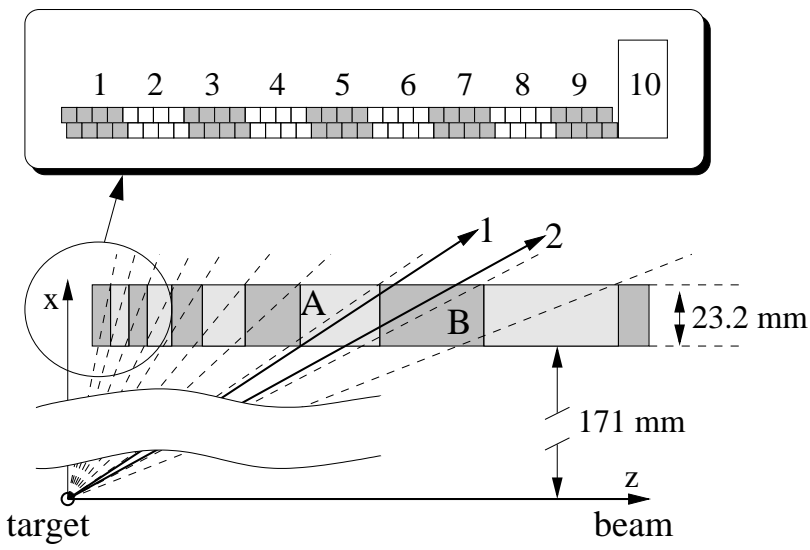

Fig. 2. Cross sectional view of adjacent scintillator bars (a) and semi-rings (b), crossed by two proton trajectories which can be distinguished by the fractional light output of elements A and B. The first 9 semi-rings are made up of scintillating fibers as shown in the figure.

overlap ensures that each charged particle originating at the target deposits energy in two adjacent bars when traversing the layer. The fractional light output from adjacent scintillator bars is used in the offline analysis (see Sect. 3.5) to give the azimuthal angle to a precision about five times better than would be possible on the basis of granularity alone [19].

The outer layer is composed of scintillator rings for angles $\theta_{\text {lab }} \leq 52^{\circ}$, split into left and right semi-rings to allow radial readout of the scintillation light in direction $\pm y$. After total reflection by $90^{\circ}$ to the top or bottom of the detector, the light is collected via light-guides by Hamamatsu R1450 photomultipliers. The width of the rings varies along the beam (cf. Fig. 2(b)) 


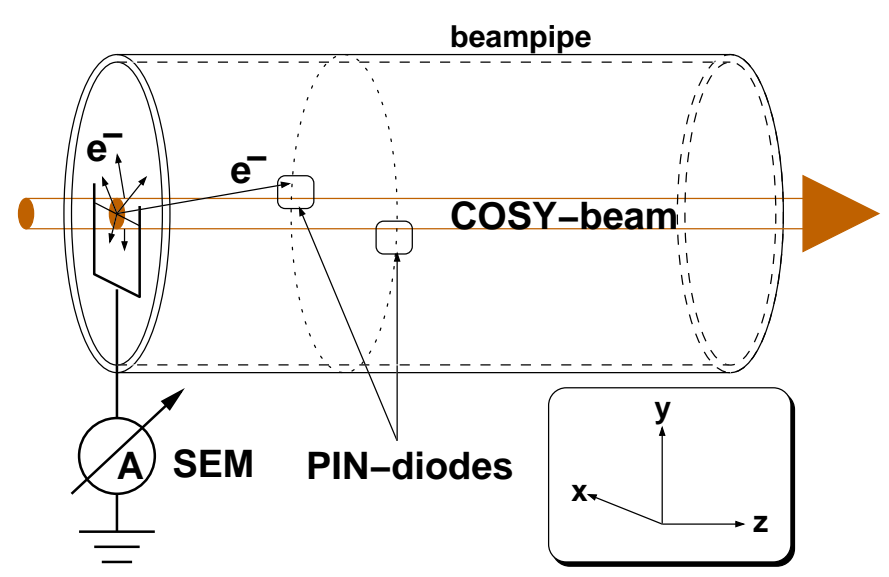

Fig. 3. Schematic setup of luminosity monitors.

such that each proton trajectory crosses 2 , in some cases 3 rings. Each ring covers an interval $\Delta \theta_{\text {c.m. }} \approx 5^{\circ}$ in the centerof-mass frame for elastic pp scattering. Again the ratio of light outputs from adjacent rings is used to improve the resolution in (polar) angle over granularity. The method becomes less and less effective with increasing polar angle. Therefore, the scintillator rings were replaced by a double layer of $\left(2 \times 2 \mathrm{~mm}^{2}\right.$ quadratic) scintillating fibers for angles $\theta_{\text {lab }} \geq 52^{\circ}$ as shown in the inset of Fig. 2] (b) [20]. The latter cover about $10 \mathrm{~cm}$ of the inner layer close to the target, and they give - by their granularity - about the same angular resolution as is obtained from the rings using fractional light output analysis.

\subsection{Luminosity Monitors}

Two independent, concurrent methods were applied to monitor luminosity as sketched in Fig. 3 Both monitors detect electrons emerging from the fiber target due to the beam-target interaction. The secondary electron monitor (SEM) consists of a fast amperemeter [21] measuring continuously the current of electrons from ground to target, replacing low-energy secondary electrons emanating from the target surface. Highenergy $\delta$-electrons from elastic proton-electron scattering were detected in two PIN diodes located at $\theta_{\text {lab }} \approx 40^{\circ}$ behind thin $\left(250 \mu \mathrm{g} / \mathrm{cm}^{2}\right)$ aluminum windows in small pockets of the beam pipe. Both rates scale differently with projectile momentum and must be corrected for this dependence when monitoring the luminosity consistently over the whole momentum range, see Sect. 3.9

\subsection{Cyclic operation}

Data are collected during synchrotron acceleration such that a complete excitation function is measured in each acceleration cycle. A typical cycle is shown in Fig. 4 After injection of some $10^{7}$ protons with $p=275 \mathrm{MeV} / c$, acceleration starts with a momentum ramp of $1.15 \mathrm{GeV} / c$ per second. At $p \approx$ $0.7 \mathrm{GeV} / c$, the target is moved vertically into the beam. The target remains in the beam until the maximum beam momentum of $p \approx 3.3-3.4 \mathrm{GeV} / c$ is reached (flattop). Since the horizontal beam position is not constant during acceleration the

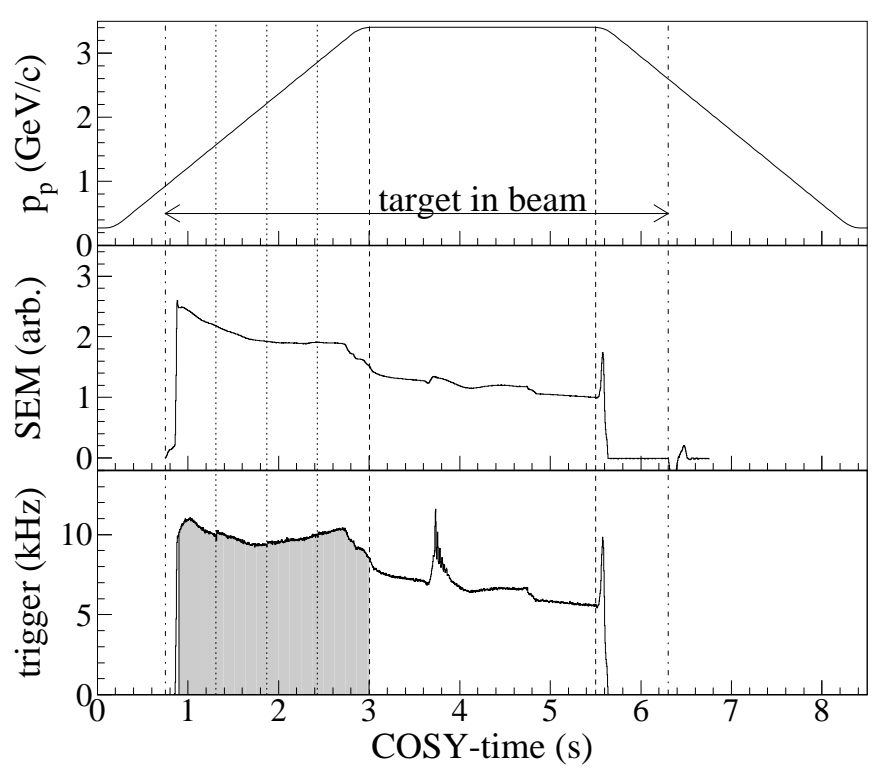

Fig. 4. Timing during an experimental cycle. Shown is the projectile momentum $p$ (top), signal of the SEM luminosity monitor (middle) and trigger rate (bottom) as a function of cycle time.

beam was steered across the fiber in the flattop (cf. Fig. 6), such that any effect relating to the position along the fiber target could be studied offline. During deceleration the beam is lost and the synchrotron prepared for the next cycle. The SEM signal indicates that the luminosity roughly remains constant during beam acceleration since beam losses are compensated by the increased beam-current when the revolution-frequency raises from 0.5 to $1.5 \mathrm{MHz}$. It should be noted that the beam emittance growth due to small angle scattering in the fiber target was nearly compensated by the adiabatic damping due to beam acceleration [22]. During the flattop the luminosity decreases with a lifetime in the order of 5-10 s.

After retraction of the target, a laser monitor system (cf. next section) is activated for $2 \mathrm{~s}$ in the flatbottom part of the cycle. For calibration and beam diagnostics, alternative cycle modes are applied, e.g. measurements at fixed momenta with longer flattop for detector calibration, cf. Sect. 3.5

\subsection{Laser Monitor System}

The EDDA-detector was monitored continuously by a laser monitor system. Light pulses are generated at a rate of $20 \mathrm{~Hz}$ by a nitrogen laser which drives a dye laser. This produces light pulses with mean wavelength $(\lambda=425 \mathrm{~nm})$ and pulse shape characteristics similar to those generated in the scintillators by real particles. They are fed through individual fibers into the light guides of all scintillator elements to illuminate all phototubes simultaneously for an online detector control. Pulse-topulse variations in laser intensity are accounted for by normalization to photodiodes receiving a constant fraction of the dye laser light. In offline analysis the laser events are used to detect long term gain drifts or jumps between detector calibration runs and to deduce necessary corrections. 
For very low energy ejectiles the produced light may exceed the linear range of the PM-response. To this end, separate laser runs are performed with the pulse intensity being stepwise decremented by a set of calibrated optical filters. By comparison to the photodiode reference the functional dependence of the digitized collected charge from the PM on the light intensity is mapped out. Offline these correction functions can be used to linearize the response of all photo-tubes individually ([23, 24] and Sect. 3.5].

\subsection{Trigger and Data Acquisition (DAQ)}

The structure and granularity of the scintillator hodoscope reflect the signature of pp elastic scattering events, namely (i) coplanarity

$$
\left|\phi_{2}-\phi_{1}\right|=180^{\circ}
$$

and (ii) kinematic correlation of scattering and recoil angle, viz

$$
\tan \theta_{\mathrm{lab}, 1} \cdot \tan \theta_{\mathrm{lab}, 2}=\frac{1}{\gamma_{\mathrm{c} . \mathrm{m}}^{2}}
$$

where $\gamma_{\text {c.m. }}=\sqrt{1+T_{p} / 2 m_{p} c^{2}}$ denotes the Lorentz factor of the pp center-of-mass motion as a function of beam kinetic energy $T_{p}$. Both conditions are used to define a fast online trigger. The coplanarity, Eq. 11, is verified by requiring a coincidence of two scintillator bars just opposite to each other with respect to the beam. Light attenuation along the bars is taken care of by adding the readouts from either end. The different flight and light transport times as well as electronic constraints require a sufficiently wide $(70 \mathrm{~ns})$ coincidence gate. Monte Carlo simulations confirm that the width of the bars guarantees a trigger even for deviations of the vertex position by as much as $\pm 1.5 \mathrm{~cm}$ from the nominal COSY beam axis.

The trigger condition for elasticity, Eq. (2), correlates each semi-ring on one (e.g. left) side of the beam with one or more on the opposite (e.g. right) side. These correlations, however, depend on projectile energy $T_{p}$ as well as the transverse profile of the beam-target overlap. If the range of rings allowed in coincidence is sufficiently wide to be valid for the whole momentum range covered during beam acceleration only a poor suppression of quasi-elastic scattering in the carbon-nuclei is achieved. For this reason programmable logic modules have been used to implement the coincidence, such that they can be reprogrammed within $5 \mathrm{~ms}$ while the beam is accelerated. A gain in trigger efficiency by a factor of two (cf. Fig. 5) was achieved by reprogramming three times during acceleration, sacrificing $1 \%$ of data acquisition time. The times when the reprogramming occurred was changed frequently to avoid empty spots in the final excitation functions.

Data are acquired whenever the target is in the beam or the laser system is triggered. Data are processed with conventional CAMAC modules for timing, pulse-height and logic signals in conjunction with a VME based event-builder. An event is written on tape when Eqs. (1) and (2) are fulfilled by at least one pair of scintillator bars and semi-rings, respectively. In addition, each event contains the cycle time from a COSY clock controlling the accelerator operation, the collected charge from all scintillator elements and the timing signals from both ends of the scintillator bars.

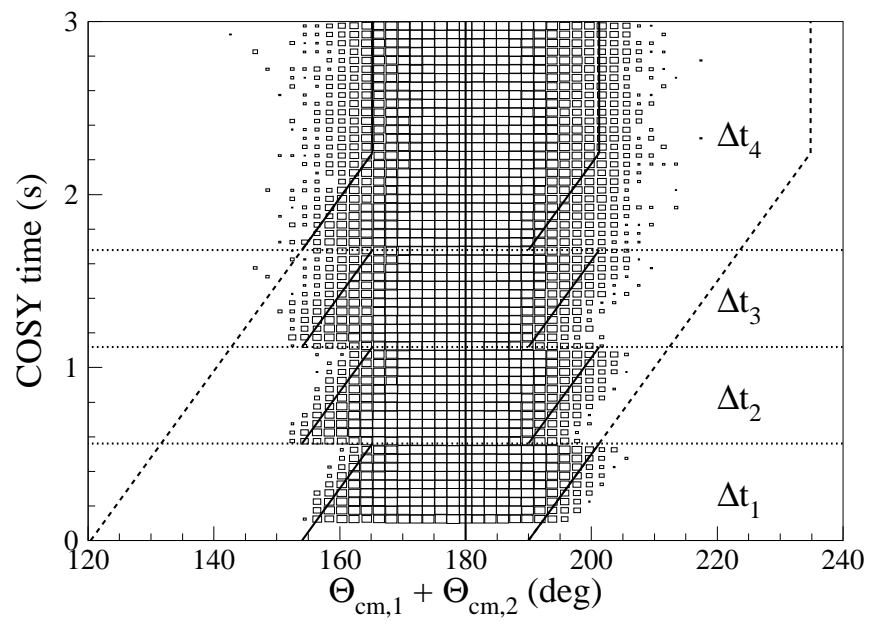

Fig. 5. Scatterplot of events taken with a carbon target during acceleration. By threefold reprogramming (dotted horizontal lines) of the kinematic trigger, the reduced acceptance (solid lines) suppresses uncorrelated background that cannot be avoided with a fixed setting (dashed lines).

There are three additional trigger sources, namely for PIN and SEM luminosity monitors and for the laser system, which are merged with the main trigger. All four independent trigger sources are counted, together with a $10 \mathrm{MHz}$ clock and pulses counting the instantaneous frequency $\nu_{\mathrm{RF}}$ of the COSY cavities, in scalers which are read out in time increments of $2.5 \mathrm{~ms}$. Comparing the number of stored events with the scaler of the corresponding trigger source yields the dead time fraction $\tau(p)$ of the data acquisition system as a function of beam momentum (i.e. cycle time) that can be corrected for in beam momentum intervals as narrow as $3 \mathrm{MeV} / \mathrm{c}$ (cf. Sect. 3.11). Our measurements were performed with time-averaged luminosities of typically $5 \cdot 10^{29} \mathrm{~cm}^{-2} \mathrm{~s}^{-1}$ and singles rates of up to $40 \mathrm{kHz}$.

\section{Offline analysis}

\subsection{Data Samples}

The data were collected in two production runs of two and three weeks, separated by half a year, with a yield of about $15 \cdot 10^{6}$ and $22 \cdot 10^{6}$ elastic events, respectively. After publication of the results based on the first production run [14], small modifications concerning e.g. the treatment of artefacts due to the detector granularity have been implemented in the analysis, advanced methods to reduce non-elastic background were developed, and a correction for radiation damage of the $\mathrm{CH}_{2}$ target introduced. Furthermore, the multitude of different running conditions, have allowed additional consistency checks and both data sets have been (re-)analyzed for the present work.

First, the data have been divided in 17 so-called data samples, which have been analyzed separately. Each sample comprises typically $1.5 \ldots 4 \cdot 10^{6}$ elastic events and consists of two data sets, one taken with a $\mathrm{CH}_{2}-$ and a $\mathrm{C}-$ target each. The relative luminosity of the $\mathrm{CH}_{2}$ - and $\mathrm{C}$-target data, measured interleaved by changing targets every few hours, was chosen online 
to yield about the same statistics for proton-carbon scattering events. Each data sample contains data

1. from only one $\mathrm{CH}_{2}$ - and one C-target,

2. with exactly the same trigger setting (e.g. reprogramming times)

3. and the same detector and COSY-beam setup.

By treating the individual results for these samples as separate measurements many consistency checks could be made prior to combining them to the final results (see Sect. 3.12).

\subsection{Overview}

For $N_{p p}$ detected elastic scattering events within a solid angle bin $\Delta \theta_{\text {c.m. }}$. and a momentum bin $\Delta p$, centered at $\left(p, \theta_{\text {c.m. }}\right)$, the differential cross section is given by

$$
\frac{d \sigma}{d \Omega}\left(p, \theta_{\text {c.m. }}\right)=\frac{N_{p p}\left(p, \theta_{\text {c.m. }}\right)}{\Delta \Omega \cdot \eta\left(p, \theta_{\text {c.m. }}\right) \cdot L_{H}(p) \cdot(1-\tau(p))}
$$

Here, $L_{H}$ denotes the absolute luminosity and $\tau$ the DAQ dead time fraction for the respective momentum bin. The detection efficiency $\eta$ accounts for the elastic scattering events discarded by the trigger or offline cuts, mainly due to secondary reactions of scattered protons in the experimental setup (Sect. 3.7.

For elastic proton-proton scattering we detect two protons and a rough selection of events is based on detector multiplicities. To this end, we subdivide the data in event classes based on the number of hits in the scintillator elements of the hodoscope and keep only those of low multiplicity. The scattering angle $\theta_{\text {c.m. }}$. is reconstructed from the vertex position and the interception points of the two trajectories, determined from the energies deposited in the scintillator elements. The vertex position moves along the target fiber during beam acceleration. Applying a kinematic fit, it can eventually be determined eventwise. The corresponding concepts are described in Sect. 3.5 and were applied to measurements with both types of target fibers.

For background reduction, a combination of an event-wise selection based on Eqs. (1) and (2) and a statistical subtraction of the carbon fiber data, normalized to the same luminosity, is accomplished in a way that - in first order - inelastic pp interactions are corrected for as well. Details are given in Sect. 3.6. For all excitation functions, the relative luminosity is provided by the two luminosity monitors. The determination of the $a b$ solute luminosity $L(p)$ in this experiment is not as accurate as in some high-precision external experiments, so that we normalize all excitation functions at one single momentum $p$ to published data (cf. Sect. 3.9.

\subsection{Monte Carlo simulations}

Experiment and analysis are accompanied by a comprehensive Monte-Carlo simulation including the details of detector geometry and materials. Particles are tracked through the detector, taking into account energy and angular straggling as well as hadronic and electromagnetic secondary interactions. Results of Monte-Carlo studies enter mainly threefold into the analysis:
1. Calculating the efficiency $\eta$ for accepting elastic pp-scattering events, this includes effects of the trigger as well of all software cuts used in the analysis.

2. For modeling the contribution of inelastic pp-reactions in order to estimate the background contribution to accepted pp-elastic events.

3. To calculate the detection efficiency of the PIN-diodes for $\delta$-electrons used for measuring the relative luminosity.

For electromagnetic interactions we use EGS4 [36] and for hadronic reactions MICRES [25] or phase-space distributions as event generators. In MICRES a number of event generators are used: To model elastic scattering cross sections distributions according to recent phase-shift analysis [7] are generated. Inelastic scattering of hadrons on nucleons and nuclei for energies up to $5 \mathrm{GeV}$ are described by tracking the impinging nucleon through the nucleus. Secondary particles including pions and hadrons are traced and their interactions with nucleons are described with known, interpolated or estimated cross sections. Momentum, energy and charge are fully conserved. In this intranuclear cascade the nucleon-nucleon interactions are either quasi-elastic or inelastic, which is modeled by exciting resonances, which may subsequently decay and eventually produce mesons. All nuclear resonances contributing more than $2 \%$ to the total inelastic cross section are taken into account. Details of this event generator MICRES are given elsewhere [25].

\subsection{Multiplicity Cuts}

For a first classification of an event, a group of $n$ hits in adjacent scintillator bars or semi-rings is called a cluster of size $n$. Due to the geometrical overlaps (cf. Fig. 2) cluster sizes $n=2$ and 3 dominate. An event can then be characterized by its cluster pattern $\left(\mathrm{N}_{B}, \mathrm{~N}_{L}, \mathrm{~N}_{R}\right)$, where $\mathrm{N}_{B}$ is the multiplicity (i.e. the number) of clusters in the layer of scintillator bars, and $\mathrm{N}_{L}\left(\mathrm{~N}_{R}\right)$ denotes the respective multiplicity for the left (right) group of semi-rings. An ideal elastic pp event reveals the pattern (211). The majority of elastic scattering events is of this type. There are, however, several patterns of higher cluster multiplicity that originate from elastic scattering due to e.g. secondary reactions in the scintillator hodoscope, accompanying $\delta$-electrons or random coincidences. By comparing the $\mathrm{CH}_{2}$ and $\mathrm{C}$-data sets the patterns containing non-negligible numbers of elastic events could be identified and we kept eight patterns, namely (211), (311), (411), (221), (212), (321), (322) and (222). For a projectile energy $T_{p}=1.5 \mathrm{GeV}$, these patterns exhaust $98.4 \%$ of all triggered events, with the ideal (211) pattern already contributing $89.2 \%$. All other patterns are discarded.

\subsection{Trajectory and vertex reconstruction}

EDDA primarily detects the position of the protons on cylinders surrounding the beam pipe. The coordinate along the circumference $R \cdot \phi$ is determined from the scintillator bars (with $R=16.4 \mathrm{~cm}$ ) and the $z$ position along the beam by the semirings. The resolution can be enhanced considerably beyond that given by the detector granularity by evaluating the charge-integrated photomultiplier-signal $S$ of all scintillator elements, usu- 
ally two, hit by the particle. This can be related to the pathlength within the scintillator element $\Delta x$ and to the average specific energy loss along this pathlength $\overline{(\mathrm{d} E / \mathrm{d} x)}$ by

$$
S\left(\theta_{\mathrm{lab}}, \phi\right)=G \cdot \overline{\left(\frac{\mathrm{d} E}{\mathrm{~d} x}\right)} \cdot \Delta x
$$

For most projectile energies and scattering angles $\theta_{\text {lab }}$ covered in the experiment, the energy loss can be considered constant along the whole path through adjacent, overlapping scintillator elements. In this simple case the gain factor $G$ describes the conversion of deposited energy to the electronic signal and encompasses (i) light transportation and attenuation in the scintillator and light guide, and (ii) photomultiplier (PM) response. The former effect introduces a dependence on the particle's point of incidence $G\left(\theta_{\text {lab }}, \phi\right)$ which is mapped out experimentally by pp elastic scattering data taken at fixed momentum $p=2.7 \mathrm{GeV} / c$, where the detector acceptance is at its maximum.

For the latter, a laser-light (cf. Sect. 2.4) fed into the light guides was used to deduce corrections for long-term gain drifts and nonlinearities close to the upper limit of the PM's dynamic range.

The spatial resolution in $\phi\left(\theta_{\mathrm{lab}}\right)$ can then be enhanced beyond the granularity given by the number of scintillator bars (semi-rings), by interpolating the point of incidence using the pulse heights $S_{1}, S_{2}$ of neighboring scintillators traversed by the charged particle. The ratio

$$
Q_{12}=\frac{S_{2} / G_{2}-S_{1} / G_{1}}{S_{2} / G_{2}+S_{1} / G_{1}}
$$

varies according to Eq. (4) monotonically with $\Delta x_{2}-\Delta x_{1}$ between +1 and -1 along the interval of overlap, see Fig. 2 Details of the algorithm are given in [19]. The resolution obtained for an ejectile is improved by a factor $\approx 5$ to $\delta \phi \approx 1.9^{\circ}$ (FWHM) and $\delta \theta_{\text {c.m. }} \approx 1.0^{\circ}$ (FWHM).

For ejectile energies below $400 \mathrm{MeV}$ two corrections are important: First, the specific energy loss $\mathrm{d} E / \mathrm{d} x$ can no longer be considered constant along the path of a particle. Assuming that the particle is an elastically scattered proton, so that the kinetic energy is known and taking into account the stopping power of all material in front of the detector element under consideration a correction can be deduced. Secondly, the amount of produced light is affected by nonlinearities in PM response. Therefore, it is linearized using information obtained with the laser monitor system (cf. Sect. 2.4). With these corrections a good angular resolution is maintained except for the lowest energies, where systematic deviations of the reconstructed position arises which will be treated as a systematic error.

In order to obtain the scattering angle the reaction vertex must be known. The vertical and longitudinal position are fixed by the fiber position and are thus the same (within a few $\mu \mathrm{m}$ ) for all events. The horizontal vertex position is smeared out by the width of the COSY beam and should be determined for each event separately. As a first step the first moments of the vertex distribution, e.g. the mean values $\left(x_{v}, y_{v}, z_{v}\right)$ and the horizontal width $\delta x_{v}$ are deduced from the data, by exploiting the coplanarity of elastically scattered protons with the beam or the kinematic correlation.

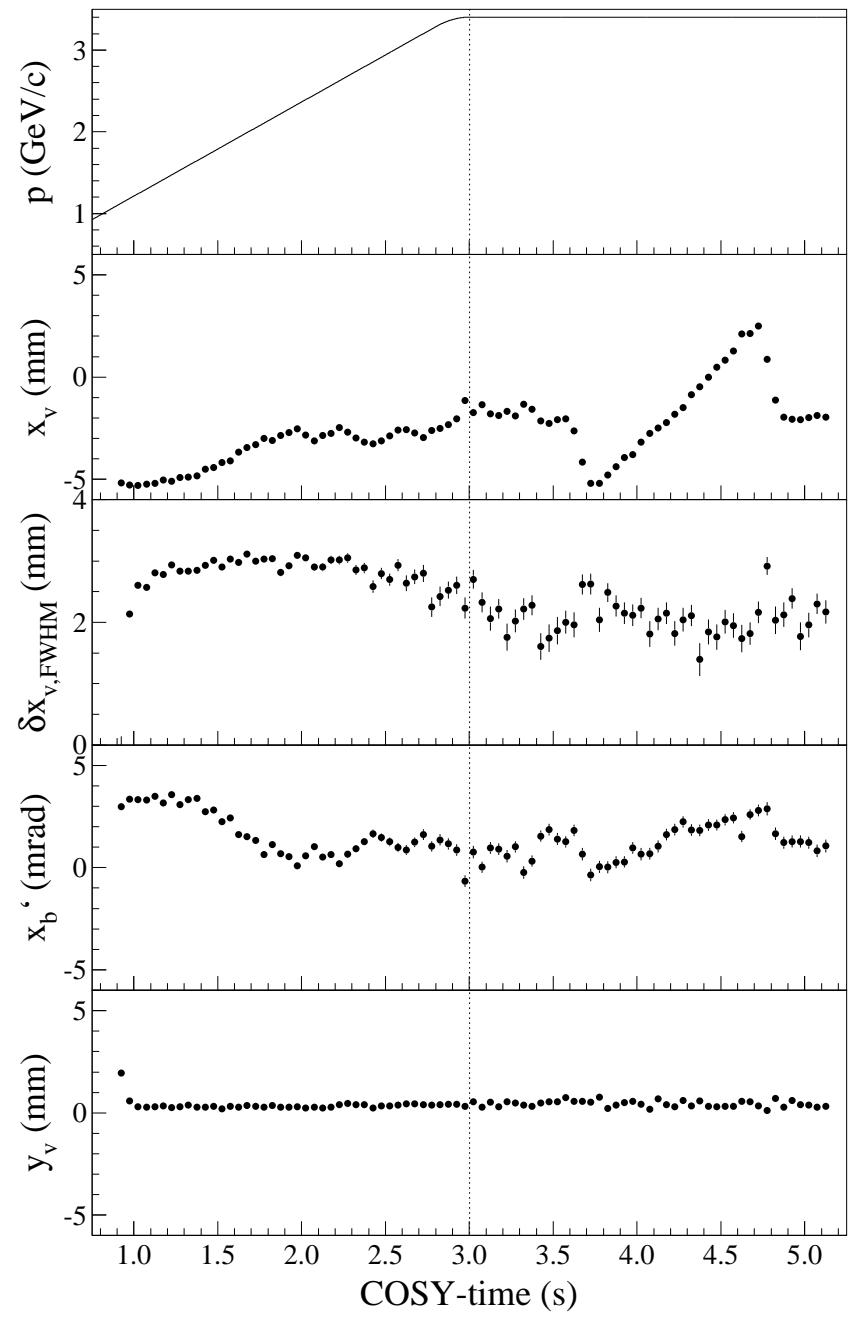

Fig. 6. Variation of horizontal vertex parameters and vertical beam position $y_{v}$ as a function of cycle time. The vertical line shows the begin of the flattop where the beam is swept on purpose horizontally over the fiber target. $x_{v}$ is the beam position, $\delta x_{v}$ the beam width folded with the detector resolution, $x_{b}^{\prime}$ the horizontal beam angle and $y_{v}$ the vertical target position. The first two points in $y_{v}$ show the settling of the target after it has been moved in.

The vertex position $x_{v}, y_{v}$ in the plane perpendicular to the symmetry axis of the detector can be determined from the pairs of points where the prongs intercept the scintillator bars. Due to the coplanarity of two body interactions the projection of the line connecting the two points into this plane must cross the vertex. The elastic scattering events are evenly distributed in $\phi$; the coordinates $x_{v}, y_{v}$ are obtained as parameters from a fit to all events for a given projectile momentum interval $\Delta p$ (details are given in [26]). The method can be extended to projectile beams tilted against the detector axis. By comparing events with equal $\left(\theta_{\text {c.m. }} \approx 90^{\circ}\right)$ or different $\left(\theta_{\text {c.m. }} \ll 90^{\circ}\right)$ lab scattering angles yields the directions $x_{b}^{\prime}$ and $y_{b}^{\prime}$ as well (cf. Fig. 6. The validity of this method was tested and verified with a beam of fixed momentum that was steered in a controlled way horizontally $(x)$ along the target fiber $(3.6 \mathrm{~s}<t<4.8 \mathrm{~s}$ in Fig. 6). The horizontal vertex position and the tilting angles of 
the beam turned out to vary during acceleration to a non negligible extent. These parameters, however, showed a remarkable long term stability over many days. Finally, the vertex position $z_{v}$ in beam direction is calculated from the interception coordinates $z_{1}, z_{2}$ in the ring-layer by making use of the kinematic relation Eq. (2) with $\gamma_{\text {c.m. being known from the instantaneous }}$ projectile momentum and $\tan \theta_{\mathrm{lab}, \mathrm{i}}=R / z_{i}$, where $R$ is the mean radius of the semi-ring layer. This way, the vertex coordinates $x_{v}, y_{v}, z_{v}$ are determined with an accuracy $\pm 0.5 \mathrm{~mm}$; the width $\delta x_{v} \approx 3 \mathrm{~mm}$ (FWHM) reflects the horizontal beam width folded with the experimental resolution.

With the mean vertex position and the position of the hit in the detector known, the polar and azimuthal scattering angles are calculated, taking into account the small correction due to the tilted beam. With the reconstruction algorithm described above, however, the horizontal vertex position cannot be determined eventwise. To improve the resolution of the final c.m. polar scattering angle $\theta_{\text {c.m. }}$, it is taken from a kinematic fit, constraint by elastic scattering kinematics (i.e Eqs. (1) and (2)). In the fit $x_{v}$ is treated as a free parameter; its distribution is compatible with the previous result for $\delta x_{v}$.

For events with higher multiplicities, i.e. pattern other than 211 , the ambiguities when correlating hits from the bar and the ring layer can be resolved almost entirely by the time-difference of the signal read out at the down- and upstream sides of the scintillator bars, which yields a position information along the bar of about $5 \mathrm{~cm}$ (FWHM) and must match the position of the struck rings. If ambiguities remain, the combination of hits which best matches elastic scattering kinematics, as outlined in the next section, is selected.

\subsection{Event Selection and Background Subtraction}

For each event we have at least two $(i=1,2)$ reconstructed tracks with angles $\left(\theta_{\text {lab }, i}, \phi_{i}\right)$ that are now subject to additional cuts to separate elastic pp scattering events from inelastic reactions or background originating from proton-carbon reactions. For this purpose one first calculates the c.m. scattering angle using pp elastic scattering kinematics:

$$
\theta_{\text {c.m. }, \mathrm{i}}=2 \arctan \left(\gamma_{\text {c.m. }} \tan \theta_{\text {lab }, \mathrm{i}}\right) ;
$$

Note that $\phi=\phi_{\text {lab }}=\phi_{\text {c.m. }}$. For elastic events both particles should be emitted back-to-back in the center-of-mass system. The angular deviation $\alpha$ from this perfect $180^{\circ}$ correlation, subsequently called the kinematic deficit, is shown in Fig. 7 for $\mathrm{CH}_{2}-$ and $\mathrm{C}$-target data at two different beam momenta. The kinematic deficit is used twofold: First, for the few events with more than two prongs the combinatorial ambiguity is removed by selecting the combination with minimum $\alpha$. The resulting probability distributions $N(p, \alpha)$ show that elastic pp events clearly stand out on a smooth, monotonic background. A comparison to the distribution obtained with a pure carbon fiber target under identical experimental conditions shows that this background can essentially be associated with quasi-free scattering on bound protons and inelastic p-C interactions. A display of events more closely related to the conditions of coplanarity, Eq. (1), and elasticity, Eq. (2), is shown in Fig. 8] where the elastic peak extends about two orders of magnitude above
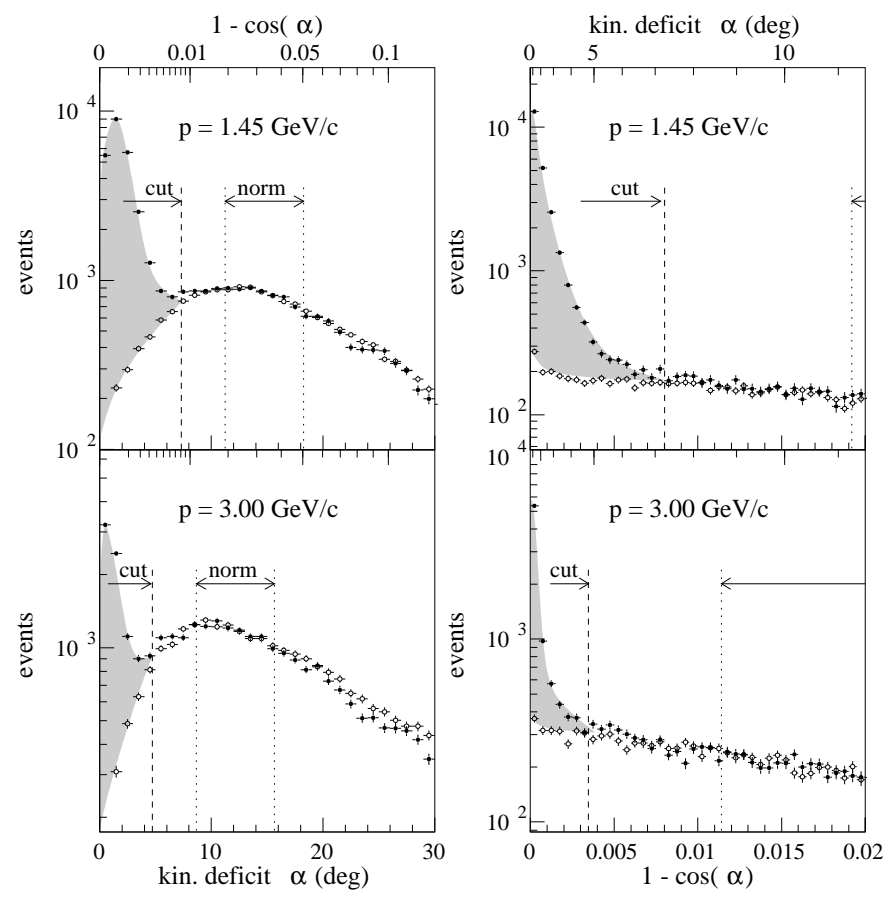

Fig. 7. Distribution of the kinematic deficit $\alpha$ for two beam momenta. Shown are events from $\mathrm{CH}_{2}$ (closed) and C-targets (open symbols). The elastic pp events are indicated by the shaded area. The upper limit $\alpha_{\text {cut }}$ (cut) for accepted elastic scattering events and the range (norm) used for normalizing the $\mathrm{C}$-target data to the $\mathrm{CH}_{2}$-data are shown. The solid angle associated with $\alpha$ rises with $\cos \alpha$, so that the number of events at very small $\alpha$ drops. The figures on the right show that indeed the elastic peak is at $\cos \alpha=1$ as expected. Note the logarithmic scale.

the background. However, the combination of those two requirements in one variable, $\alpha$, is more effective for quantitative background treatment.

In a first step, a projectile momentum dependent cut $\alpha \leq$ $\alpha_{\text {cut }}(p)$ is applied in a way that - based on Monte-Carlo simulations and comparisons to the C-target data - almost no elastic events are discarded. The remaining events are sorted into two-dimensional arrays $N_{\mathrm{CH}_{2}}\left(p, \theta_{\text {c.m. }}\right)$ and $N_{\mathrm{C}}\left(p, \theta_{\text {c.m. }}\right)$ with the option to adjust the bin-widths $\Delta p$ and $\Delta \theta_{\text {c.m. }}$. to statistical requirements.

\subsubsection{Statistical Background Subtraction}

Background subtraction is performed statistically: A factor $\mathcal{L}(p)$ is deduced from the normalization in the interval $\alpha_{\min }=\alpha_{\text {cut }}+$ $4^{\circ} \leq \alpha \leq \alpha_{\text {cut }}+11^{\circ}=\alpha_{\max }$ of the $\alpha$-distribution $N_{\mathrm{CH}_{2}}(p, \alpha)$ of a $\mathrm{CH}_{2}$-target data set to that $N_{\mathrm{C}}(p, \alpha)$ of the associated $\mathrm{C}$-target data set, i.e.

$$
\mathcal{L}(p)=\frac{\left.N_{\mathrm{CH}_{2}}(p)\right|_{\alpha_{\min } \leq \alpha \leq \alpha_{\max }}}{\left.N_{\mathrm{C}}(p)\right|_{\alpha_{\min } \leq \alpha \leq \alpha_{\max }}}
$$

where events have been integrated over all scattering angles. $\mathcal{L}$ can be viewed as the luminosity with respect to proton-carbon scattering of the $\mathrm{CH}_{2}$-target relative to the $\mathrm{C}$-target data set. Its 


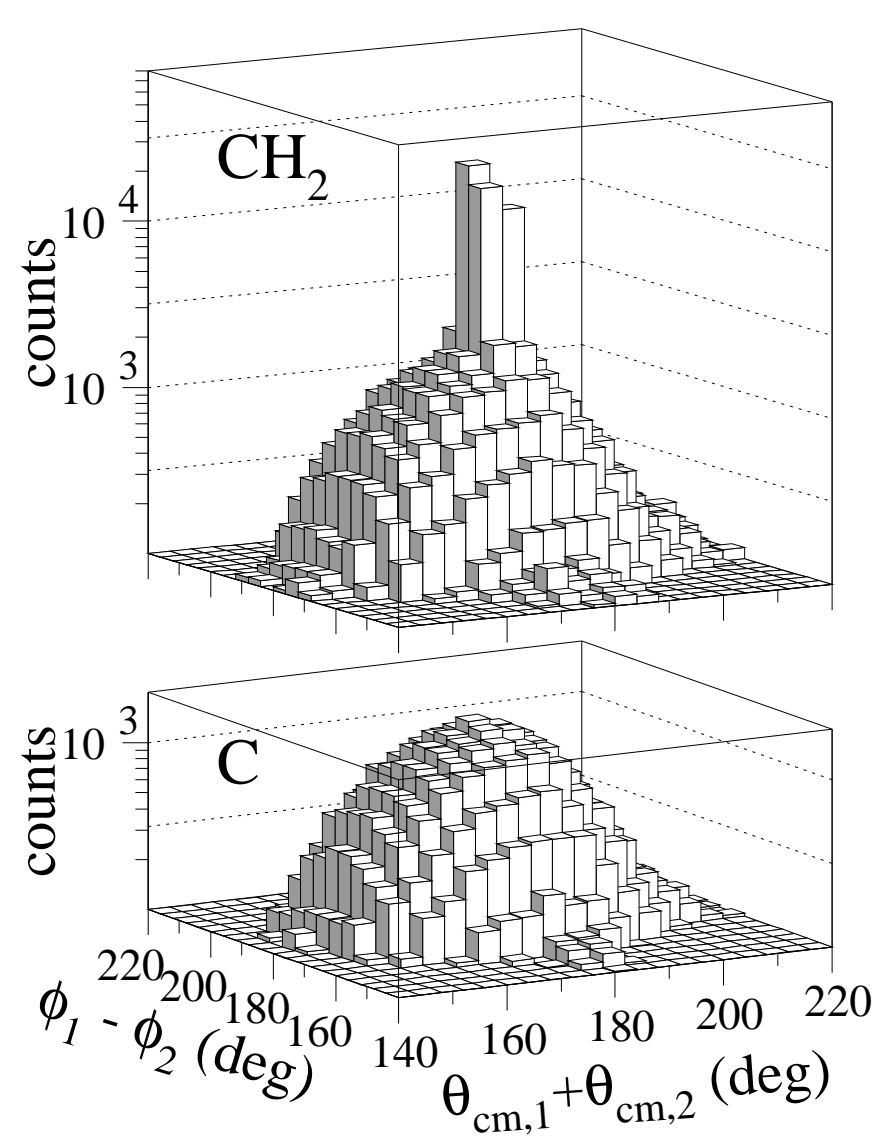

Fig. 8. Distribution of angle integrated events obtained with a $\mathrm{CH}_{2}$ (top) and carbon (bottom) fiber target at $p=2.25 \mathrm{GeV} / c$. Note the logarithmic scale.

momentum dependence is caused by the different COSY beam lifetimes for the two targets due to their different thickness. $\mathcal{L}$ was determined for all contributing multiplicity patterns separately, to account for slightly modified contributions from accidental coincidences caused by the luminosity difference. The background can now be subtracted statistically viz

$$
N_{\text {pp }}\left(p, \theta_{\text {c.m. }}\right)=N_{\mathrm{CH}_{2}}\left(p, \theta_{\text {c.m. }}\right)-\mathcal{L}(p) \cdot N_{\mathrm{C}}\left(p, \theta_{\text {c.m. }}\right)
$$

There are two concerns when applying this method: first, the $\mathrm{CH}_{2}$-targets are aluminum-coated and it must be verified that $\mathrm{p}-\mathrm{C}$ and $\mathrm{p}-\mathrm{Al}$ reactions in this region of phase-space are indistinguishable. This was proven by taking data sets with plain and aluminum coated $\mathrm{C}$-targets. The carbon-subtracted pp elastic scattering yields is the same within statistical errors. Applying the statistical subtraction scheme to $\mathrm{C}+\mathrm{Al}-$ and $\mathrm{C}$-target data sets (instead of $\mathrm{CH}_{2}$ and $\mathrm{C}$ ) produced results for " $N_{\mathrm{pp}}$ " compatible with zero. A second concern is that inelastic protonproton scattering populates the $\alpha$-distribution in the region where $\mathcal{L}$ is determined and will therefore systematically distort the results. To shed light on this issue Monte-Carlo simulation were performed to mimic the effect of inelastic pp reactions.

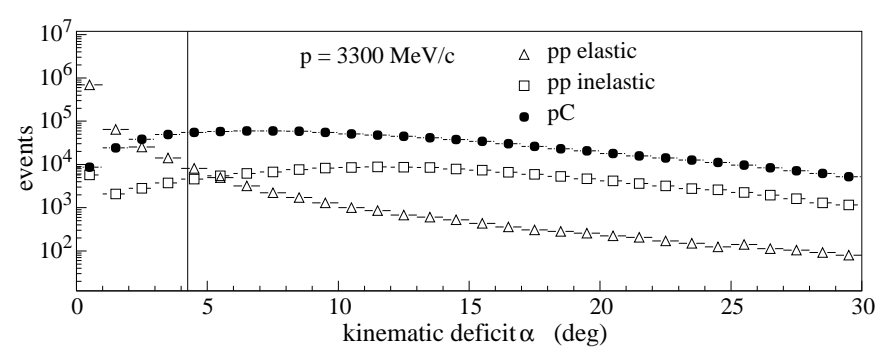

Fig. 9. Simulated distributions of the kinematic deficit $\alpha$ for $\mathrm{p}-\mathrm{C}$ reactions (solid dots), elastic (triangles) and inelastic (squares) pp scattering. The vertical line indicates the position of $\alpha_{\text {cut }}$.

\subsubsection{Contributions of Inelastic pp-Reactions}

Elastic scattering exhausts at least half the pp total cross section in the COSY energy range. Except for $\mathrm{pp} \rightarrow \mathrm{d} \pi^{+}$all inelastic reactions have at least 3 particles in the final state and therefore the probability to look like an elastic event is expected to be small. Since these reactions cannot be measured separately by our experiment we rely on Monte-Carlo (MC) simulations, using the available information on the respective total cross sections. Unfortunately, phase-space distributions are practically unknown so that models have to be used in MC event generation. For this purpose distributions either according to phase-space or generated by MICRES, where inelastic reactions proceed through excitation of resonances, are used.

Simulations show that the main inelastic contribution originates from the channels $\mathrm{pp} \rightarrow \mathrm{pn} \pi^{+}, \rightarrow \mathrm{pp} \pi^{+} \pi^{-}$and $\rightarrow \mathrm{pp} \pi^{\circ}$. The cuts on the cluster pattern and the correlation mismatch $\alpha$ reduce this background to less than $1.5 \%$ of the (angle integrated) elastic yield, whereas the corresponding number for the $\mathrm{p}-\mathrm{C}$ background is up to one order of magnitude higher; both increase with beam momentum $p$.

Their simulated $\alpha$-distributions are shown in Fig. 9 for the worst case, i.e. at high momenta. Both background distributions are similar in shape. Therefore, the statistical subtraction with its normalization performed in the angular range $\alpha \geq \alpha_{\text {cut }}$ takes care of the main part of pp inelastic events with $\alpha \leq \alpha_{\text {cut }}$, too. A closer look, however, reveals differences in shape that will give rise to a systematic background error which has been studied in more detail.

For this purpose, $N_{\mathrm{CH}_{2}}\left(p, \theta_{\text {c.m. }}\right.$. ) has been numerically composed from three contributions, namely

$$
N_{\mathrm{CH}_{2}}=N_{\mathrm{pp}}^{\text {el. }}+N_{\mathrm{pp}}^{\text {in. }}+N_{\mathrm{C}}^{(\mathrm{a})}
$$

where the elastic contribution $N_{\mathrm{pp}}^{\mathrm{el}}$ is based on our experimental results [14]. The second term is Monte Carlo generated and the third is an experimental data sample $N_{\mathrm{C}}^{(\mathrm{a})}$ obtained with a carbon target, properly normalized to the observed ratio of pp to $\mathrm{p}-\mathrm{C}$ events. This set is then corrected for background with the statistical method, Eq. 8), by using a second, statistically independent carbon measurement $N_{\mathrm{C}}^{(\mathrm{b})}$. From the result the term $N_{\mathrm{pp}}^{\text {el. }}$ used before in Eq. (9) is subtracted. The remaining difference $D\left(p, \theta_{\text {c.m. }}\right)$ reflects the incorrect treatment of the inelastic pp component $N_{\mathrm{pp}}^{\mathrm{in}}$, and turns out to be systematically 


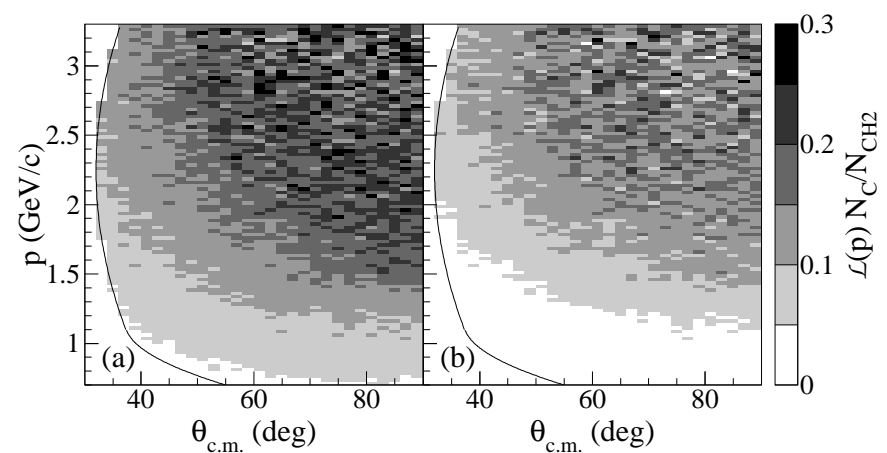

Fig. 10. (a) Ratio of events from carbon- and $\mathrm{CH}_{2}$-targets of one data sample after all cuts have been applied and the carbon data have been properly normalized. The solid line shows the acceptance cut on the c.m. scattering angle. In (b) the same data are shown when in addition event selection using fuzzy-logic methods is utilized.

positive, indicating that the inelastic pp contribution was insufficiently subtracted as is expected from Fig. 9 The fraction $\left|D / N_{\mathrm{pp}}^{\mathrm{el}}\right|$ is negligible (i.e. less than $1 \%$ ) except for the largeangle $\left(\theta_{\text {c.m. }}>70^{\circ}\right)$ and high-momentum $(p>2.5 \mathrm{GeV} / c)$ region where the contribution may reach about $5.5 \%$. Because only the total cross sections but not the angular distributions of most inelastic pp reactions are sufficiently well known, no attempts have been made to correct this deviation and the maximum effect obtained with different models is taken as a systematic uncertainty.

\subsubsection{Hydrogen Absorbed in Carbon-Fibers}

A close look at the distribution for events measured with carbonfiber targets reveals a tiny peak at the locus of elastic pp scattering, cf. Fig. 8 which could be due to traces of hydrogen or water absorbed in the carbon fiber. Indeed does this peak show an angular distribution in agreement with the expectation for elastic pp scattering. The background subtraction following Eq. (8) will then reduce the number of elastic pp events of the $\mathrm{p}-\mathrm{CH}_{2}$ measurement systematically wrong. A careful study of this reduction showed that it is for all targets smaller than $0.5 \%$ and does not vary with projectile momentum. Therefore, it is equivalent to a reduction of the effective $\mathrm{CH}_{2}$ target thickness and its effect cancels completely when finally all excitation functions are normalized at one momentum to some precise reference cross-section, cf. Sect. 3.9.4

\subsubsection{Statistical Errors}

The statistical error associated with the background subtraction is roughly given by $\delta N_{\mathrm{pp}}=\sqrt{N_{\mathrm{CH}_{2}}+\mathcal{L}^{2} N_{\mathrm{C}}}$ where the error of $\mathcal{L}$, which is a minor contribution, has been left out for the sake of simplicity. Thus any contribution from scattering off carbon that can be eliminated by cuts prior the subtraction

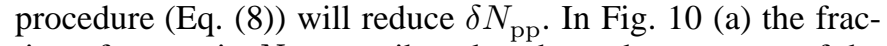
tion of events in $\mathrm{N}_{\mathrm{CH}_{2}}$ attributed to the carbon-content of the target is shown. It rises with momentum and angle from 5\% up to $25 \%$ and unfortunately is largest where scattering rates are the lowest. Therefore, it was investigated how the statistical method could be complemented by an eventwise classification scheme to reduce $N_{\mathrm{C}}$ prior to being subtracted. To this end information on each event besides the measured angles, like energy losses, can be used. When testing methods commonly employed in pattern-recognition, like self-organizing maps or artificial neural networks, we found an approach based on socalled fuzzy-logic to be particularly helpful. It allowed a large reduction of the number of proton-carbon scattering events to be subtracted statistically as shown in Fig. 10](b), and will be discussed in detail in the next section.

\subsubsection{Background subtraction using Fuzzy-Logic}

So far only the information on the proton angles have been used to identify elastic scattering events. In addition, the EDDA detector provides energy-loss information in the bars and semirings and timing information from the bar layer, which can be put into use to test their compatibility with elastic pp-scattering events. However, quasi-elastic scattering from nucleons in the carbon-nucleus as well as inelastic pp reactions are not cleanly separated in these variables, so that simple cuts are insufficient. Here, methods developed within the framework of so-called "fuzzy-logic" [27, 28] have been shown to be very powerful [29].

For each event, described by a vector $\boldsymbol{q}$ of $n$ measured or reconstructed quantities $q_{i}(i=1 \ldots n)$, we must construct a decision-function $d(\boldsymbol{q})$ to decide if it is accepted $(d(\boldsymbol{q})=1)$ or not $(d(\boldsymbol{q})=0)$ as a candidate for an elastic scattering event. When using simple cuts on one or two-dimensional projections of $\boldsymbol{q}$ applied in sequence most of the correlations between the observables $q_{i}$ are lost, since one variable outside one cut is sufficient to discard an event. In the fuzzy-logic approach we define for each observable $q_{i}$ a so-called member-function $P_{i}$ : $q_{i} \mapsto[0,1]$, which maps the parameter space of $q_{i}$ onto the interval between zero and one. It is designed such, that events with a high (low) probability of being elastic yield values $p_{i}$ close to $1(0)$. For this purpose the member function must not be a probability in the strict sense and we use

$$
P_{i}\left(q_{i}\right)=1-\frac{N_{\mathrm{C}}\left(q_{i}\right)}{N_{\mathrm{CH}_{2}}\left(q_{i}\right)} .
$$

To obtain $P_{i}$ with sufficient statistical precision, the data are binned in $q_{i} . N_{\mathrm{C}}$ and $N_{\mathrm{CH}_{2}}$ are the number of events within a certain bin centered at $q_{i}$ for this quantity, whereas all other quantities $q_{j}$ with $i \neq j$ may have arbitrary values. Note, that here and in the following it will be assumed that the $\mathrm{CH}_{2}$-and $\mathrm{C}$-target data samples have been properly normalized to the same luminosity.

These member-functions are then combined to a combined "probability" by the $\gamma$-operator [27, 28]

$$
P^{(g)}(\boldsymbol{q})=\left[\prod_{i=1}^{n} P_{i}\left(q_{i}\right)\right]^{1-g} \cdot\left[1-\prod_{i=1}^{n}\left(1-P_{i}\left(q_{i}\right)\right)\right]^{g}
$$

mapping the parameters space in $\boldsymbol{q}$ onto the interval $[0,1]$. It features a, yet to be fixed, parameter $g \in[0,1]$, to the effect 

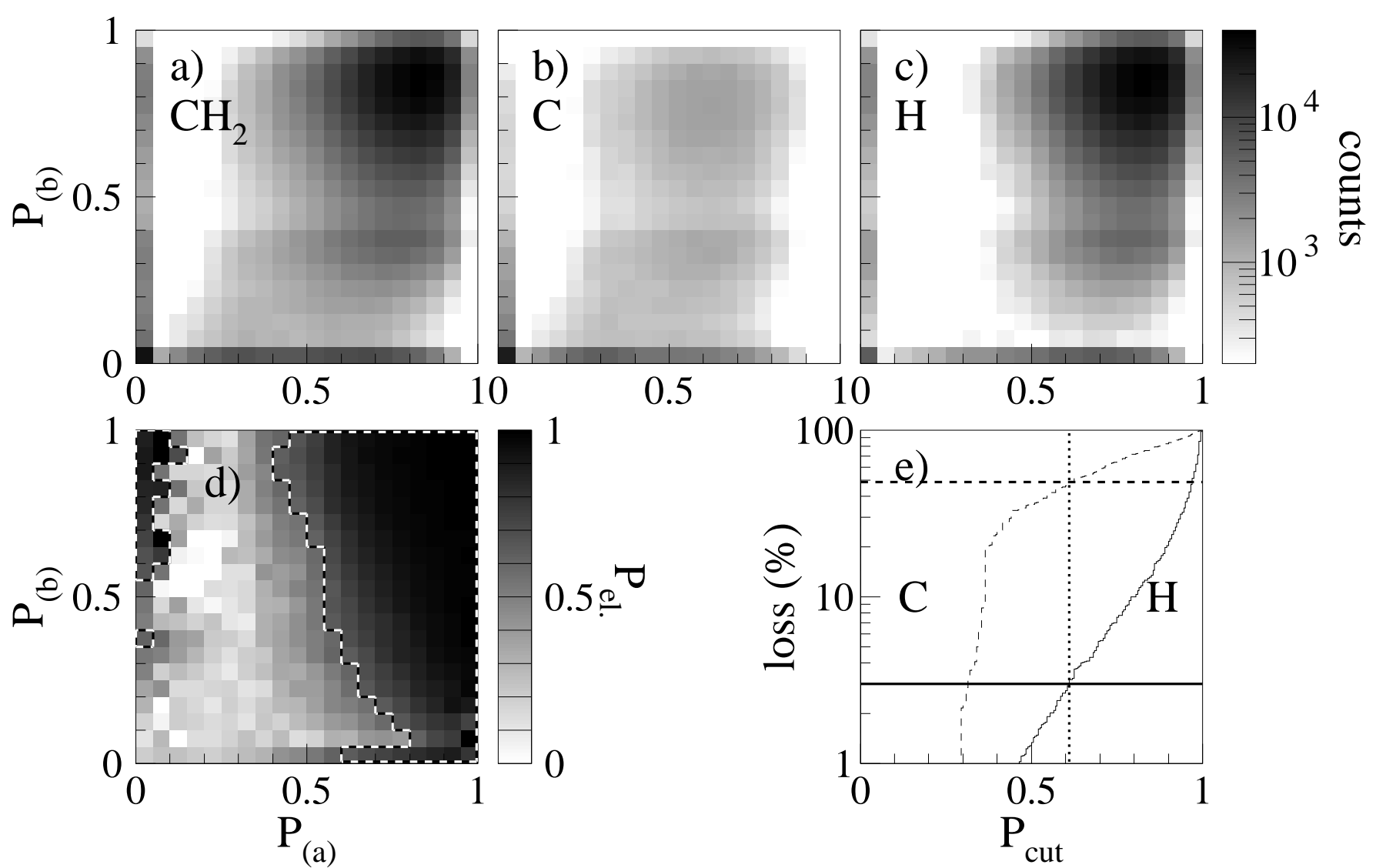

Fig. 11. Distribution of events in the $\left(P_{(a)}^{(g)}, P_{(b)}^{(g)}\right)$ plane for a $\mathrm{CH}_{2}$ (a) and C-target (b) normalized to the same luminosity. In (c) the difference attributed to pp-scattering is shown, and in (d) the "probability" $P_{\mathrm{el}}$. as defined in Eq. 13. The dashed line shows a typical contour for accepted events based on the condition $P_{\mathrm{el}} .>P_{\text {cut }}$ which corresponds to a loss of $3 \%$ of elastic events (e) while reducing the background by about $50 \%$. The spectra shown correspond to events around $p \approx 2.1 \mathrm{GeV} / c$ and are integrated over all scattering angles.

that the $\gamma$-operator specializes to the well-known logical AND $(g=0)$ or OR $(g=1)$ operators in the limiting cases. By this procedure we have mapped the information contained in $\boldsymbol{q}$ to a single variable $P^{(g)}$ between 0 and 1 .

In this experiment we used $\mathrm{n}=5$ experimental quantities $q_{i}$ :

$\alpha: \quad$ The kinematic deficit as defined in Sect. 3.6

$\Delta \phi: \quad$ The difference of the azimuthal angles of the two tracks,

$\Delta_{T z} \quad$ The correlation of the time-of-flight (TOF) $T_{1}-T_{2}$ and the $z$-position difference in the bar layer of the two charged tracks:

$$
\Delta_{T z} \equiv\left(T_{1}-T_{2}\right)-\beta_{\text {c.m. }}\left(z_{1}-z_{2}\right),
$$

where $\beta_{\text {c.m. }}=\sqrt{T_{p} /\left(2 m_{p}+T_{p}\right)}$. This quantity is close to zero for elastically scattered protons as required by kinematics.

$\Delta E_{1(2)}$ : The energy loss in the bar layer for the particle scattered to the left (right) of the beam.

These were combined to two vectors $\boldsymbol{q}_{(a)}=\left(\alpha, \Delta \phi, \Delta_{T z}\right)$ and $\boldsymbol{q}_{(b)}=\left(\Delta E_{1}, \Delta E_{2}\right)$. This allows us to map each event onto a unit square spanned by $P_{(a)}^{(g)}\left(\boldsymbol{q}_{(a)}\right)$ and $P_{(b)}^{(g)}\left(\boldsymbol{q}_{(b)}\right)$ as shown in Fig. 11] for $g=0.5$. The elastic events clearly stand out in the upper-right corner in the $\left(P_{(a)}^{(g)}, P_{(b)}^{(g)}\right)$ plane, whereas reactions on carbon - considered as background - are spread out over a larger region. Increasing the value of $g$ spreads out the background over a larger area, whereas a decrease does the same to the elastic scattering events, so that $g=0.5$ appeared to be a good compromise.

In principle one could now define the decision function $d\left(\boldsymbol{q}_{(a)}, \boldsymbol{q}_{(b)}\right)$ by drawing a two-dimensional contour on the $P_{(a)}^{(g)}$ vs. $P_{(b)}^{(g)}$ distribution. However, the signal (pp elastic scattering) and the background (p-C reactions) do overlap, so that any reduction of the latter will always be accompanied by some unavoidable loss of signal. However, in large regions of the $\left(P_{(a)}^{(g)}, P_{(b)}^{(g)}\right)$-plane the losses will be very small. This can be quantified by defining $P_{\mathrm{el}}$, again viz

$$
P_{\text {el. }}\left(P_{(a)}^{(g)}, P_{(b)}^{(g)}\right) \equiv 1-\frac{N_{\mathrm{C}}\left(P_{(a)}^{(g)}, P_{(b)}^{(g)}\right)}{N_{\mathrm{CH}_{2}}\left(P_{(a)}^{(g)}, P_{(b)}^{(g)}\right)} .
$$

If we sort the data in $20 \times 20$ bins in the $\left(P_{(a)}^{(g)}, P_{(b)}^{(g)}\right)$ plane (cf. Fig. 11 (d)) we can view $P_{\text {el. }}$ as a probability that an event falling into a certain bin is from elastic scattering. Then, it is optimal to discard events with a low $P_{\text {el. }}$, i.e. defining a deci- 


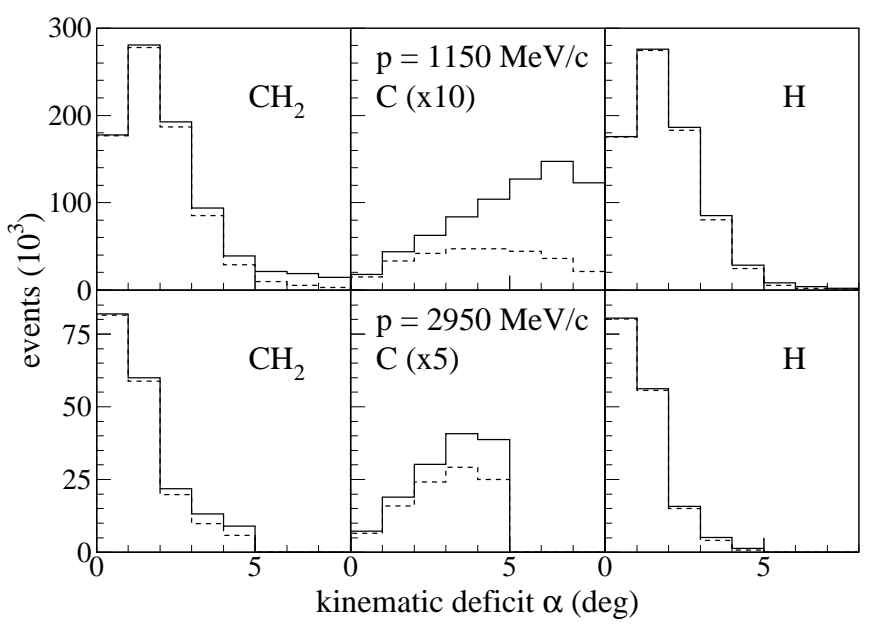

Fig. 12. Spectra of the kinematic deficit $\alpha$ for two beam momenta ranges $( \pm 150 \mathrm{MeV} / \mathrm{c})$ with (dashed line) and without (solid line) application of background rejection using fuzzy-logic. Shown are the data from $\mathrm{CH}_{2}$ - (left) and $\mathrm{C}$-targets (center, normalized to the same luminosity) as well as the difference (right) attributed to the hydrogen contents. Note that a cut at $7.5 \ldots 8^{\circ}$ (top) or $4.5 \ldots 5^{\circ}$ (bottom) on $\alpha$ has already been applied.

sion function:

$$
d(\boldsymbol{q})=\left\{\begin{array}{l}
1 \text { for } P_{\text {el. }}(\boldsymbol{q}) \geq P_{\text {cut }} \\
0 \text { for } P_{\text {el. }}(\boldsymbol{q})<P_{\text {cut }}
\end{array}\right.
$$

where $P_{\text {cut }}\left(p, \theta_{\text {c.m. }}.\right)$ is to be chosen momentum and angle dependent. Since the background originating from the carbon has been measured separately, we can now quantify both the background reduction as well as the loss of elastic events as a function of $P_{\text {cut }}$. In Fig. 111(e) we show a typical example. However, this procedure is done for every bin in $\left(p, \theta_{\text {c.m. }}\right)$ and every data sample individually. One obtains a sizeable reduction of background events $(\mathrm{C})$ for minor losses of elastic scattering events $(\mathrm{H})$. We decided to sacrifice $3 \%$ of the elastic scattering events, allowing for a reduction of the background of about $60 \%$. A typical contour of accepted events is shown as a dashed line in Fig. 11 (d). The events with $P_{(a)}^{(g)} \approx 0$ are populated mainly by events where the time information of the scintillator bars is ambiguous due to multiple hits. This occurs for some elastic events as well, so that this class had to be kept.

In order to determine $P_{\text {cut }}\left(p, \theta_{\text {c.m. }}\right)$ with high statistical precision we determined its value for $0.3 \mathrm{GeV}$ wide momentum and $10^{\circ}$ wide $\theta_{\text {c.m. }}$.bins. Its value is typically around 0.5 and smoothly varying with the beam momentum and c.m. scattering angle. When applying the cut to the final data, its value has been interpolated for the correct momentum and angle.

To summarize: by transferring the detector information by methods adopted from fuzzy-logic - to two variables with values between 0 and 1 , any event can be mapped onto the unit square. Since the data are binned we group the data in 400 classes according to their $P_{(a)}^{(g)}$ and $P_{(b)}^{(g)}$ values. Finally we select a certain subset of these classes by requiring that the respective $P_{\text {el. }}$ is above a certain threshold $P_{\text {cut }}$, chosen to result in only a small, well-defined loss of elastic events. This reduces

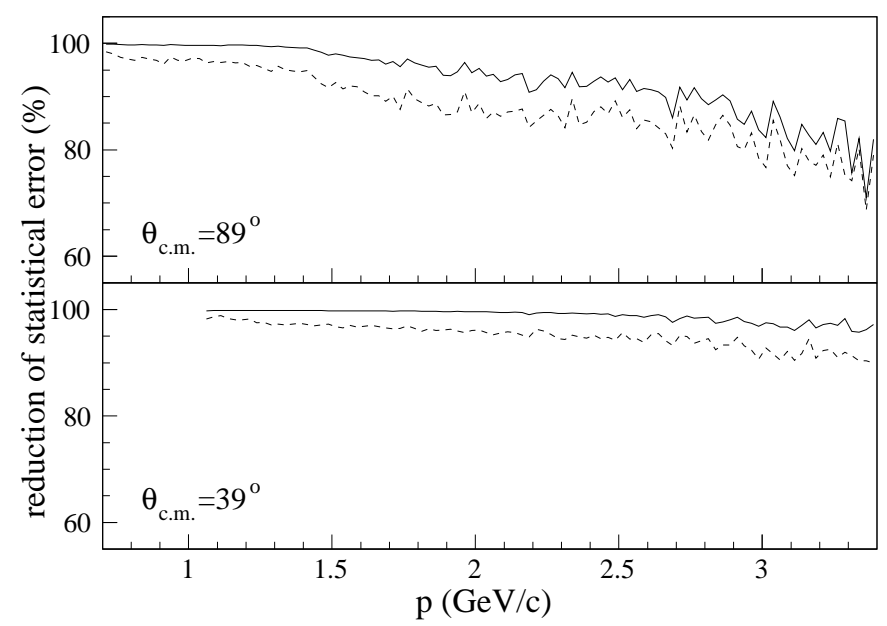

Fig. 13. Relative reduction of the statistical error from carbon subtraction when applying the cut from the fuzzy-filter approach (dashed line) and the reduction on the total uncertainty (solid line) when all other sources of errors are included.

the number of events, $N_{\mathrm{CH}_{2}}\left(p, \theta_{\text {c.m. }}\right)$ and $N_{\mathrm{C}}\left(p, \theta_{\text {c.m. }}\right)$, entering Eq. (8) to correct for the contribution arising from carboncontents of the target (Sect. 3.6.1]. Since less events are subtracted (Figs. 10 (b) and 12) the corresponding statistical uncertainty is considerably reduced (cf. Fig. 13), most notably at high momenta and large c.m. scattering angles where elastic cross sections are smallest.

Since the resulting loss of elastic events is independent of beam momentum and angle and its absolute value can be determined with $0.5 \%$ precision (e.g. $3 \% \pm 0.5 \%$ ) this is not offset by a large increase of the systematic uncertainty. To this end a comparison of the final cross sections with and without pre-selection of events using fuzzy-logic (Fig. 14) shows no systematic differences. The precise value 0.5 of the parameter $g$ introduced in Eq. (11) is not important, however, values close to 0 or 1 are to be avoided, since either the elastic events are concentrated in only a few classes or spread out over all classes. Then $P_{\text {el. }}$ can not be determined with sufficient precision for each class and the net effect on background reduction is diminished.

\subsection{Detection Efficiency}

The probability $\eta\left(p, \theta_{\text {c.m. }}\right)$ that an elastic pp-scattering event leads to a valid trigger and survives all offline cuts was determined by Monte-Carlo simulations. It uses the available information on the vertex-distribution, beam position and angles and accounts for the electronic thresholds and trigger conditions. All simulated events are then analyzed by the same software as used for the experimental data, applying the same reconstruction algorithms and cuts. The fraction of events passing these cuts $\eta\left(p, \theta_{\text {c.m. }}\right)$, is fairly constant between 0.94 and 0.96 and shows no dramatic momentum dependence. The efficiency is smallest for the lowest momenta and forward scattering and for the highest momenta and symmetric scattering, i.e. $\theta_{\mathrm{lab}, 1} \approx \theta_{\mathrm{lab}, 2}$ or $\theta_{\text {c.m. }} \approx 90^{\circ}$. The main reduction is due to secondary reactions of the ejectiles in the beam-pipe or 


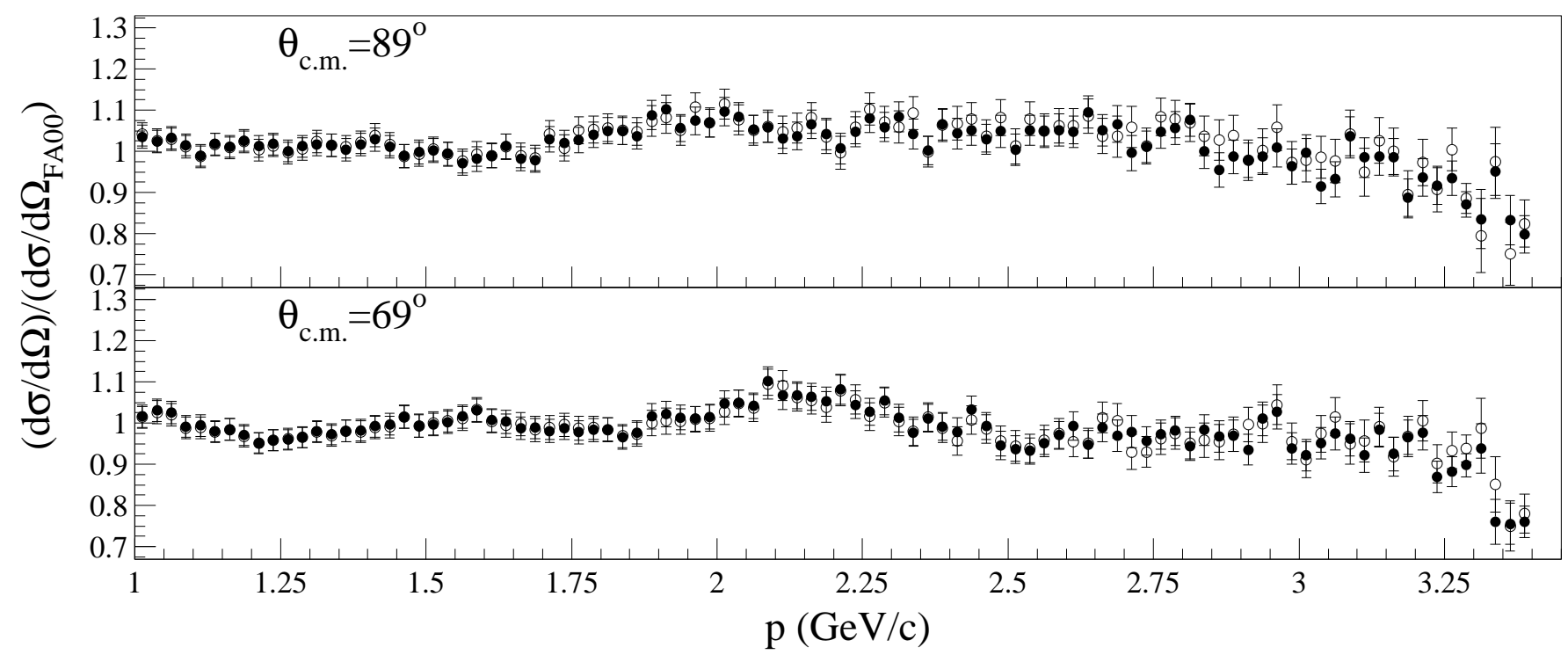

Fig. 14. Comparison of two excitation functions with (closed) and without (open symbols) using the fuzzy-filter approach for background subtraction. To remove the main energy dependence all cross sections have been divided by the predictions of the PSA-solution FA00 of [8].

the detector, so that the information on one or both protons is sufficiently distorted to be removed on the trigger-level or by offline-cuts. The loss of 4 to $6 \%$ is consistent with the expectation derived from the known total hadronic cross-sections and the thickness and composition of matter in the detector set-up. For application, $\eta\left(p, \theta_{\text {c.m. }}\right.$. $)$ is approximated by a polynomial expansion in $\cos \theta_{\text {c.m. }}$. with momentum dependent coefficients.

\subsection{Detector Acceptance}

To account for the acceptance limits of the EDDA detector two software cuts, on $\theta_{\text {c.m. }}$ and $\phi$, are applied in the analysis: The limits of the acceptance in the c.m. scattering angle $\theta_{\text {c.m. }}$ is given by the requirement that both protons are detected in the half-ring layer of the EDDA-detector. The minimum scattering angle accepted for the final data (shown as the solid line in Fig. (10) was selected large enough to rule out elastic events to be lost due to the extended beam-target overlap or small-angle scattering of the ejectiles in the beampipe and the detector.

Although the EDDA detector yields full azimuthal coverage a cut on $\phi$ is applied as well: For $\phi$ close to $90^{\circ}$ or $270^{\circ}$, events are lost on the trigger level when both particles hit the same - left or right - half of the ring layer. In addition the angular resolution achieved with the semi-rings is reduced at these angles, since close to the readout large variations in the light collection efficiency occur. Thus, we artificially reduced the azimuthal acceptance by selecting only events with the left particle emitted at $|\phi| \leq 76^{\circ}$ and accounted for this reduced acceptance with a factor $180 / 152=1.184 \pm 0.007$ in the crosssection calculation.

\subsection{Normalization}

In internal target experiments the luminosity depends on the overlap of the target density distribution with the transverse profile of the stored beam. During acceleration both the horizontal beam position and the emittance - determining the beam width - vary considerably (cf. Fig. 6) and cannot be determined, e.g. from elastic scattering data (cf. Sect. 3.5), with sufficient precision. Therefore, two electromagnetic processes with known dependence on the instantaneous luminosity and beam energy were recorded concurrently with the elastic scattering data. These are the current of secondary electrons (SEM) emanating from the fiber target which is a function of the energy deposit by the beam, and the rate of elastically scattered electrons, so-called $\delta$-electrons, in PIN-diodes placed at $40^{\circ}$ behind thin windows in the beam pipe (cf. Fig. 3). These two methods are referred to as SEM- and PIN-monitors.

As it will be outlined in the following sections, both are not accurate enough to allow an absolute luminosity measurement. However, the main uncertainties enter as multiplicative factors which are independent of beam-momentum and can be eliminated by an absolute normalization to high-precision reference data [30] at one beam momentum $\left(p_{\text {ref }}=1.455 \mathrm{GeV} / \mathrm{c}\right)$. The change of the luminosity with respect to this reference momentum is provided by the SEM- and PIN-monitors with high accuracy, and will be referred to as relative luminosity determination.

Furthermore, both monitors are sensitive to the electron contents of the target only and measure $L_{e}$, i.e. the luminosity with respect to proton-electron interactions. To relate this to the Luminosity $L_{H}$ for proton-proton interactions the hydrogen $\bar{\rho}$ and electron $\rho_{e}$ densities in the target enter, viz

$$
L_{H}=\frac{\bar{\rho}(p)}{\rho_{e}} L_{e}=\frac{\bar{\rho}}{\rho_{0}}(p) \cdot \underbrace{\frac{\rho_{0}}{\rho_{e}} L_{e}}_{L_{\mathrm{SEM}} \text { or } L_{\mathrm{PIN}}},
$$

where $\rho_{0}$ is the hydrogen density of an unused $\mathrm{CH}_{2}$-target. The hydrogen density $\bar{\rho}$ is the weighted average over the region of the target sampled by the COSY-beam and therefore 
momentum dependent. Its value will gradually decrease due to radiation damage in the course of the experiment and will be discussed in Sect. 3.9.3 For convenience the factor $\rho_{0} / \rho_{e}$ has been included in $L_{\mathrm{SEM}}$ and $L_{\mathrm{PIN}}$, so that it is fixed by the absolute normalization and the factor $\frac{\bar{\rho}}{\rho_{0}}(p)$, common to both monitors, is deduced separately. A corresponding, but much smaller change in the electron density will also be taken into account.

\subsubsection{Secondary Electron Monitor (SEM)}

The SEM rate is proportional to the average energy $\overline{\Delta E} d e$ posited in the target fiber [31], and therfore to the restricted energy loss rate $\left|\frac{\mathrm{d} E}{\mathrm{~d} z}\right|_{T<T_{\text {cut }}} \mid$ [32], which takes into account that energetic knock-on electrons above some energy $T_{\text {cut }}$ will escape from the target and not contribute to the energy deposit. We fix the cut-off energy to $9 \mathrm{keV}$ by requiring that the effective range of electrons equals half the target thickness. However, any choice within a factor of 5 yields the same energy dependence within $0.6 \%$, but not the same absolute value of the restricted energy loss. Thus, we expect the current $I_{\mathrm{SEM}}$ of emitted secondary electrons, as recorded by a sensitive amperemeter [21], to be given by

$$
I_{\mathrm{SEM}}=k_{\mathrm{SEM}} \cdot L_{\mathrm{SEM}}(p) \cdot\left|\frac{\mathrm{d} E}{\mathrm{~d} z}(p)\right|_{T<T_{\mathrm{cut}}} \mid .
$$

The current is carried by the electrons emitted from the target surface, and is dominated by electrons of very low energy, produced by thermalization of the deposited energy. Hence, the proportionality factor $k_{\mathrm{SEM}}$ should not depend on the precise initial distribution of the electron energies and thus the beam momentum - it will, however, be sensitive to the structure of the surface. Any local damage of the aluminum-coating of the fiber and a resulting possible charge-buildup would make $k_{\text {SEM }}$ a function of the position $x$ along the fiber. Since the proton beam moves along the target horizontally during acceleration, this would result in an indirect dependence of $k_{\mathrm{SEM}}$ on $p$. Such an effect has been ruled out by comparing $L_{\mathrm{SEM}}$ to the elastic pp-scattering rate when steering the beam across the target horizontally during the flattop (cf. Fig. 6), so that $p$ is fixed and only $x$ was varied.

\subsection{2 $\delta$-Electron (PIN) Monitor}

Elastically scattered electrons are detected at $40^{\circ}$ by $500 \mu \mathrm{m}$ thick PIN diodes with an area of $10 \times 10 \mathrm{~mm}^{2}$. They are mounted in pockets at $\phi=0^{\circ}$ and $180^{\circ}$ in the beampipe, behind thin $(250 \mu \mathrm{m})$ aluminum windows. The electrons are produced according to the well-known Rosenbluth cross section [33, 34] with the simplification, that due to the small momentum transfers $(\approx 1 \mathrm{MeV} / \mathrm{c})$ at COSY-energies the form-factors are unity. Detection at $40^{\circ}$ offers both statistical precision ( $\mathrm{d} \sigma / \mathrm{d} \Omega_{\mathrm{lab}} \approx$ $200 \mathrm{mb} / \mathrm{sr})$ and electrons of sufficiently large energies $(0.5-$ $1.5 \mathrm{MeV}$ ) to be observed with high efficiency.

The luminosity is determined in two steps: experimentally the rate of scattered electrons $N_{e}$ is deduced from the singles

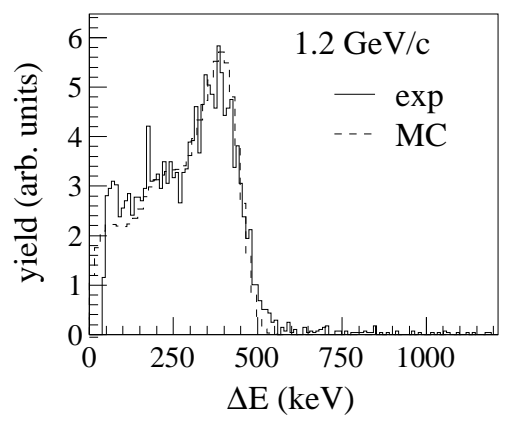

Fig. 15.

Com-

par-

i-

son

of

the

en-

ergy

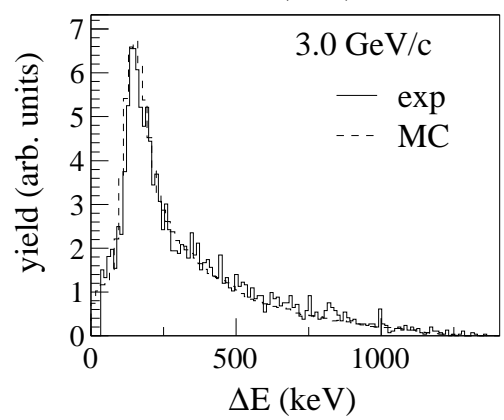

de-

posit

of

$\delta$ -

electrons

in

the

PIN

diodes

with

MC-

simulations.

rate in the PIN-diodes $N_{\text {PIN }}$, corrected for the rate of hadrons $N_{h}$.

$$
N_{e}=N_{\mathrm{PIN}}-N_{h}
$$

Since all electrons are stopped within the PIN-diodes or their mounting, the rate of hadrons is determined by looking for coincident hits in both bars and semi-rings of the outer detector layer (cf. Fig. 11) positioned behind the respective PIN-diode when viewed from the target. We found that $4-6 \%$ of all PINtriggers had to be attributed to hadrons, with the fraction linearly increasing with beam momentum. To estimate the number of hadrons escaping detection in the outer layer because of secondary reactions or stopping, a telescope was mounted in place of a PIN-diode in an independent measurement. The telescope comprised a $5 \times 5 \mathrm{~mm}^{2}$ PIN-diode followed by a $\mathrm{Cu}$-absorber, thick enough $(0.7 \mathrm{~mm})$ to stop all electrons, and a $10 \times 10 \mathrm{~mm}^{2}$ PIN-diode to detect the hadrons. It could be shown that that $0.5 \%$ of all PIN triggers not vetoed by the outer-detector layers are due to hadrons [35] and that this fraction is not beammomentum dependent.

Secondly, the number of electrons has to be related by a Monte-Carlo simulation to the luminosity (cf. Eq. 15)

$$
L_{\mathrm{PIN}}(p)=\frac{\rho_{0}}{\rho_{e}} N_{e}(p) \cdot l_{\mathrm{MC}}(p)
$$

where $\rho_{0} / \rho_{e}$ is the ratio of free protons to electrons in the target and $l_{\mathrm{MC}}$ is the average luminosity in p-e elastic scattering needed to produce one detected electron. The latter is given by MC-integration of the Rosenbluth cross-section, taking into account the exact geometry of the PIN-diodes, all materials used for mounting and shielding, the COSY-beam direction, location and width (Sect. 3.5) and the detection thresholds determined experimentally. The interaction of the the electrons were modeled using the electron-gamma shower code EGS4 [36]. In Fig. 15 the shape of the the experimental energy-loss spectrum is compared to MC-simulations, showing the transition from stopped electrons at lower energies to a typical Landau-like 


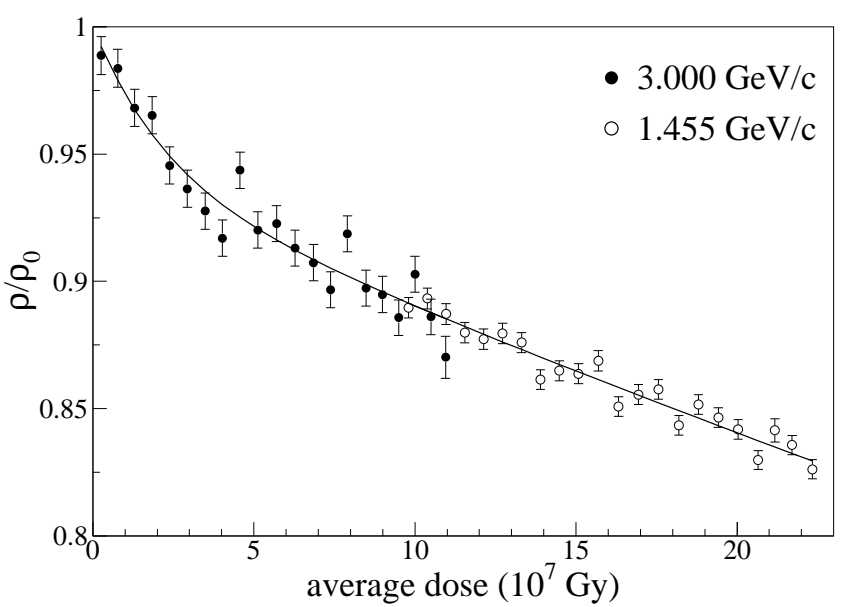

Fig. 16. Relative change of the hydrogen contents of $\mathrm{CH}_{2}$-targets as a function of the accumulated dose as determined from measurements at fixed energies. The solid line is a parameterization as outlined in the text (from [38]).

energy-loss distribution at higher energies. Note, that - apart from normalizing to the same number of counts - no parameters have been adjusted using the experimental data. The absolute energy scale was fixed by calibration with conversionelectrons from a ${ }^{175} \mathrm{Hf}$-source. The main source of systematic errors in $l_{\mathrm{MC}}$ [37] are uncertainties in

1. the target location along the beam,

2. the horizontal beam position,

3. the thickness of the aluminum window

4. and the precise size of the depleted area of the PIN-diodes.

By taking the arithmetic mean of luminosity determined with the PIN-diodes mounted to the left and right of the beam, the second errors cancel and the last errors do not contribute when looking only at changes of $l_{\mathrm{MC}}$ with beam momentum. The resulting systematic error of $L_{\mathrm{PIN}}(p) / L_{\mathrm{PIN}}\left(p_{\text {ref }}\right)$ increases with $\left|p-p_{\text {ref }}\right|$ and stays below $2.9 \%(1 \%)$ for beam momenta below (above) $p_{\text {ref }}=1.455 \mathrm{GeV} / \mathrm{c}$.

In principle an absolute luminosity could be deduced, but its precision hinges on the knowledge of the ratio of electrons to protons in the $\mathrm{CH}_{2}$-target. The naive estimate of $\rho_{0} / \rho_{e}$ being four is changed by the aluminum coating $\left(\approx 20 \mu \mathrm{g} / \mathrm{cm}^{2}\right)$ to about 4.7. In addition, radiation damage (see Sect. 3.9.3) reduces the number of hydrogen in the target, as outlined in the next section. The absolute normalization to reference data agrees within $5-10 \%$ with our estimates of $\bar{\rho} / \rho_{e}$, consistent with the errors due to uncertainties of the aluminum thickness, the size of the depleted area of the PIN diodes and the accumulated dose of a specific target.

\subsubsection{Correction for Radiation Damage of the Target}

The hydrogen density of the $\mathrm{CH}_{2}$-target decreases during the course of the experiment. This is due to radiation-induced crosslinking of the polypropylene polymers by replacing two $\mathrm{C}-\mathrm{H}$ bonds by a $\mathrm{C}-\mathrm{C}$ bond and $\mathrm{a}_{2}$ molecule emanating from the target. In comparison, losses due to hadronic interactions are

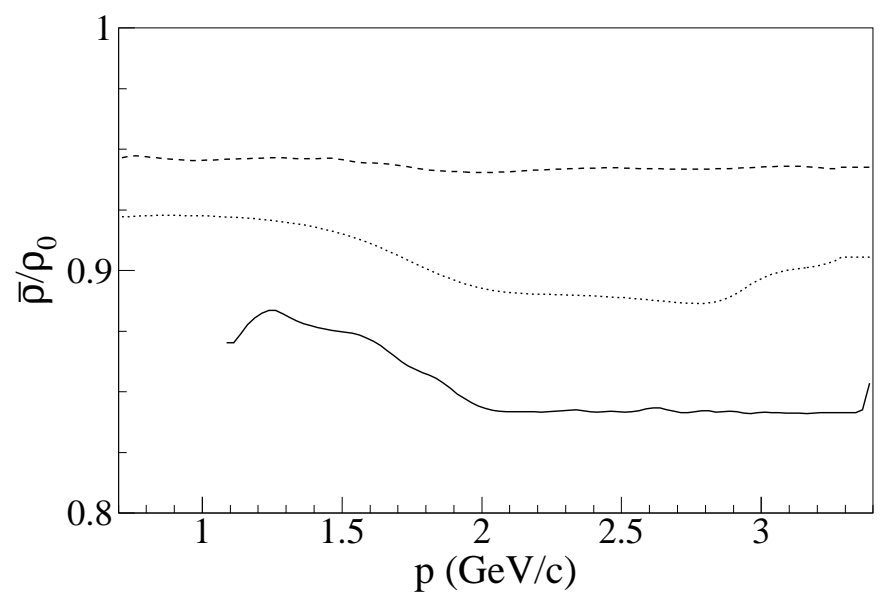

Fig. 17. Average relative hydrogen density as a function of momentum for three samples corresponding to different setups of the COSY beam.

negligible. The relative change of the hydrogen density $\rho$, and to a lesser extent of the electron density, with respect to an undamaged target $\rho_{0}$ will be a function of the acquired dose. The dose is not deposited uniformly along the target, but reflects the density distribution of the beam over the course of many COSY machine cycles (cf. Fig. 6). Finally, during the acceleration target regions with different hydrogen density will be sampled at different momenta which needs to be corrected for.

To this end the history of each target with respect to the acquired dose has to be closely monitored. The rate at which dose is acquired is related to the instantaneous luminosity $L_{H}(t)$, the specific energy loss of beam particles in the target and the normalized horizontal beam profile $P(x, t)$ viz

$$
\frac{\mathrm{d} D}{\mathrm{~d} t}(x, t)=\left.\frac{\mathrm{d} E}{\mathrm{~d} z}\right|_{T<T_{\text {cut }}} ^{(p(t))} \frac{k L_{H}(t) P(x, t)}{\rho(t) / \rho_{0}},
$$

where the constant $k=113.6 \mathrm{~Gy} \mathrm{~mm} \mathrm{mb} \mathrm{cm} / \mathrm{MeV}$ encompasses all information on target material and dimensions (see [38] for details). Since $L_{H}$ is the luminosity with reference to the hydrogen content of the target, the factor $\rho / \rho_{0}$ corrects for the average relative hydrogen contents sampled by the beam.

The relative change of the hydrogen content has been determined experimentally, by observing the relative change in the yield from pp-elastic scattering with respect to that of inelastic p-carbon reactions at constant momentum as a function of the acquired dose, calculated from the measured luminosity [38]. The result is shown in Fig. 16 together with a parameterization of the form

$$
\frac{\rho}{\rho_{0}}(D)=f \exp \left(-\lambda_{1} D\right)+(1-f) \exp \left(-\lambda_{2} D\right) .
$$

The loss is described by a fast process with decay constant $\lambda_{1}=(4.4 \pm 1.3) \cdot 10^{-8} \mathrm{~Gy}^{-1}$ which reduces the hydrogen density by at most $f=(5.8 \pm 0.7) \%$, and a slow process with decay constant $\lambda_{2}=(5.67 \pm 0.28) \cdot 10^{-10} \mathrm{~Gy}^{-1}$, with an additional scale uncertainty of $\lambda_{1,2}$ of $11 \%$.

For each target the luminosity was recorded for the time of exposure to the COSY-beam and the beam profile $P(x, t)$ is 


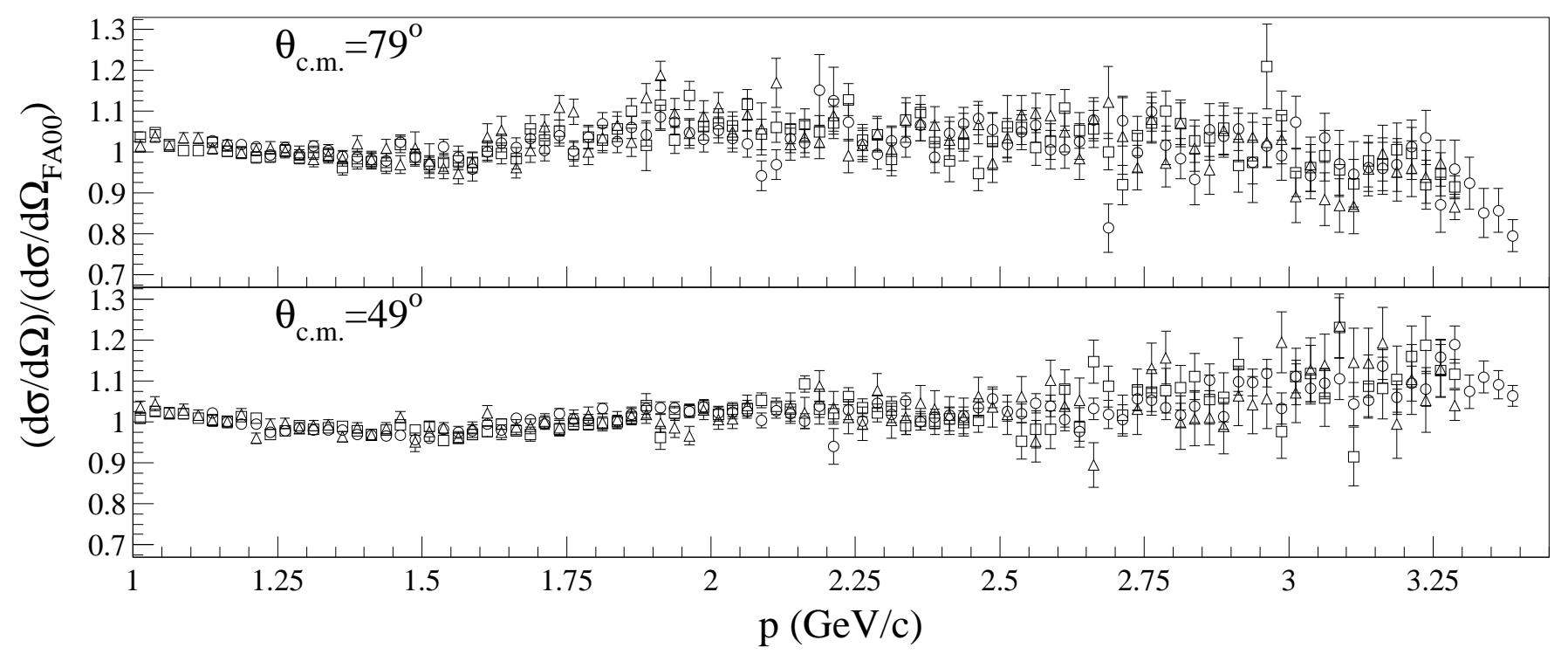

Fig. 18. Comparison of the differential cross section of the three groups of samples (distinguished by the symbols $\bigcirc, \square$, and $\triangle$ ). Only statistical errors are shown. To remove the main energy dependence all cross sections have been divided by the PSA-prediction of [8] (solution FA00).

known from the reconstructed vertex distribution (cf. Sect. 3.5) and the dose can be calculated with Eq. (19) in a first approximation by assuming $\rho / \rho_{0}=1$ to obtain the dose distribution. Using the hydrogen density as given by Eq. (20) the dose calculation can be iterated until self-consistency is reached.

Finally, the hydrogen density as a function of the beam momentum during the acceleration, averaged over the beam profile and the total time of the measurement is calculated for each data sample. Three examples, corresponding to different setups of the COSY-beam are shown. It turned out that for about one third of the data this correction is not momentum dependent (dashed line in Fig. [17), because the change of beam position was small compared to the width of the beam. For other samples the correction amounts to typically $5-15 \%$.

The shape of the correction factor only depends on the setup of the COSY beam. The data samples have been taken with three different settings, each one comprising roughly one third of the data. In Fig. 18 excitation functions obtained with these three beam setups are compared at two c.m. scattering angles, showing perfect agreement within statistics. When these cross sections are integrated over the full detector acceptance of the detector, they agree much better than the $2.5 \%$ error corridor, as given by the relative luminosity determination.

Note, that the PIN-monitor is affected indirectly by changes in electron density $\rho_{e}$ of the target (cf. Eq. (18)). However, most of the electrons are attached to the carbon and aluminum nuclei and the applied correction is smaller by a factor of about 4.8. The SEM monitor is not affected by cross linking, since the SEM-yield is determined by the electron density in the aluminum coating of the target.

\subsubsection{Absolute Normalization}

The SEM and PIN monitors record the change of the luminosity with beam momentum very accurately. The absolute value is obtained by normalizing the pp-elastic scattering angular distribution at one projectile momentum bin $p_{\text {ref }}$ to precise reference data of differential cross sections. We use the precision measurement at $p_{\text {ref }}=1.455 \mathrm{GeV} / c\left(\mathrm{~T}_{\mathrm{ref}}=793 \mathrm{MeV}\right)$ by Simon et al. [30], where the absolute normalization uncertainty is given as $\leq 1 \%$. By numerically integrating the data points of [30] we arrive at a reference cross section

$$
\begin{aligned}
\sigma_{\text {ref }} & =2 \pi \int_{39.84^{\circ}}^{88.51^{\circ}} \mathrm{d} \theta_{\text {c.m. }} \sin \theta_{\text {c.m. }} \frac{\mathrm{d} \sigma_{\text {ref }}}{\mathrm{d} \Omega_{\text {c.m. }}}\left(\theta_{\text {c.m. }}\right) \\
& =11.16 \pm 0.02^{\text {stat. }} \pm 0.11^{\text {syst. }} \mathrm{mb} .
\end{aligned}
$$

to be used for the final absolute normalization. By numerically integrating the angular distribution at $p_{\text {ref }}$ of this experiment over the same angular range, the values of $k_{\mathrm{SEM}}$ and $\rho_{0} / \rho_{e}$ (Eqs. (16) and (18) are adjusted to yield the same integrated elastic scattering cross section $\sigma_{\text {ref }}$.

The absolute normalization receives an additional statistical error from the statistical precision of the angular distribution of our data samples at the normalization momentum $p_{\text {ref }}$. The procedure to minimize the influence of this error on the final result when combining all data samples is described in Sect. 3.12

\subsubsection{Comparison of Luminosity Monitors}

In Fig. 19 the result for the integrated luminosity from the SEM- and PIN-monitors are compared for one typical data sample. The agreement is typically much better than $2.5 \%$. The error on the luminosity derived from e-p elastic scattering (PINmonitor) is dominated by systematic uncertainties from the MCsimulations. Furthermore, below $1.1 \mathrm{GeV} / c$ the $\delta$-electrons at $40^{\circ}$ are so low in energy, that they can no longer be detected by the PIN-monitor. Therefore the luminosity obtained from 


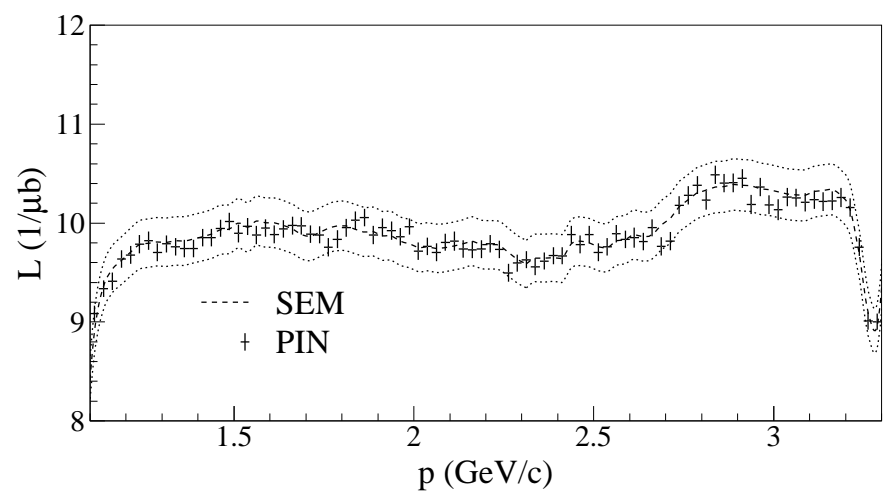

Fig. 19. Time-integrated luminosity for one out of 17 data samples from the two luminosity monitors. The dashed line shows a $\pm 2.5 \%$ band around $L_{\mathrm{SEM}}$ to test the consistency to $L_{\mathrm{PIN}}$. The absolute normalization scale has been fixed at $p_{\text {ref }}=1455 \mathrm{MeV} / c$. Note the suppressed zero on the vertical axis.

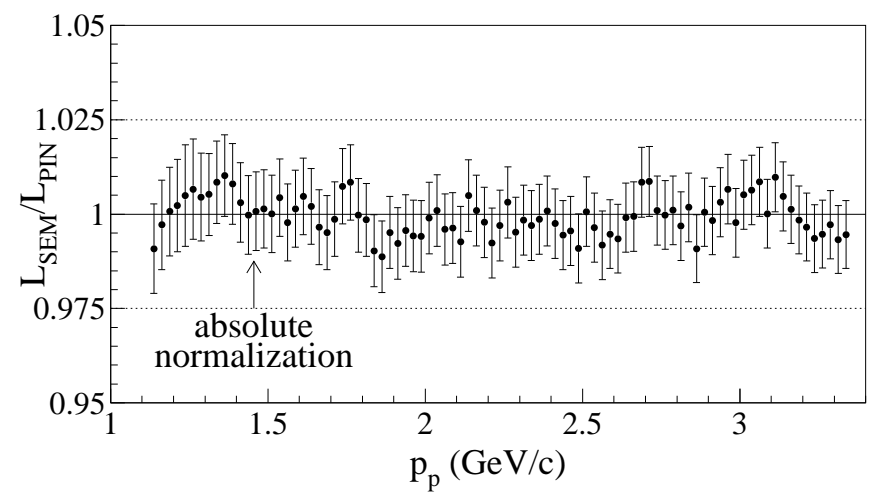

Fig. 20. Comparison of the SEM and PIN luminosity monitors averaged over all data samples. The errors are dominated by the uncertainty in $l_{\mathrm{MC}}$.

the SEM-monitor is used in the analysis and the PIN-monitor is taken as a consistency check and to derive an error estimate on the relative luminosity determination. When averaged over all data samples the two luminosity monitors give consistent results (cf. Fig. 20) and deviations stay below $1.25 \%$ within known statistical and systematic errors. Since some samples show deviations as large as $2.5 \%$ we use this value as an error estimate of the systematic relative luminosity determination. This corridor is shown in Figs. 19 and 20 as dotted lines.

The total absolute luminosity summed over all data samples is shown in Fig. 21 The first data taking period covered the momentum range 1.1-3.4 $\mathrm{GeV} / \mathrm{c}$ and the second one 0.7$3.3 \mathrm{GeV} / c$. The spikes close to the maximum energy are due to the reduced ramping speed in the transition to the flattop of the accelerator cycle. Note that the luminosity is more or less constant over the whole momentum range (cf. Figs. 19] and 21. This shows that the loss of beam particles due to the fibertarget is compensated by the increase in beam current due to the rising revolution frequency during acceleration. This is in line with the finding [22, 39], that the heating of the stored beam by the fiber-target is counterbalanced by the adiabatic damping of the betatron oscillations evinced as a near-constant beam-width $\delta x_{v}$ (cf. Fig. 3.5.

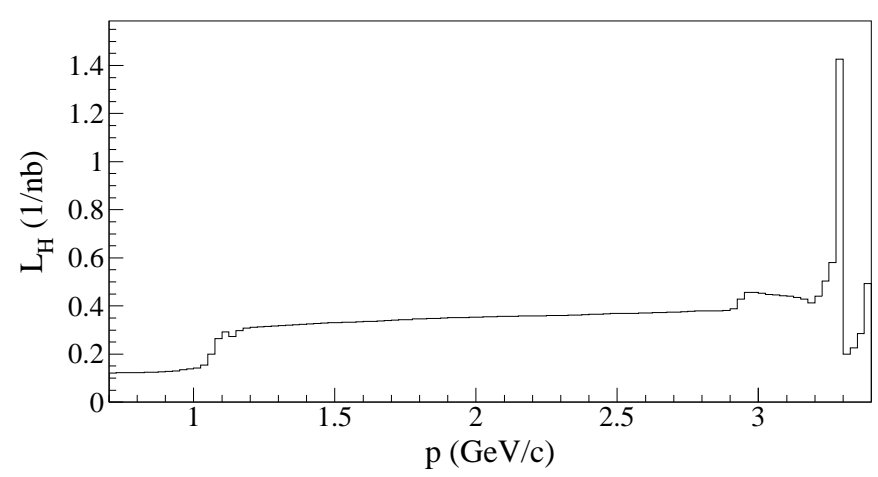

Fig. 21. Total integrated luminosity for all data.

\subsection{Beam Momentum Determination}

The beam momentum for each scattering event is determined from the relative time $t$ of the event with respect to the start of the COSY-cycle. This allows to calculate the nominal momentum $p(t)$ of the COSY-beam based on the mathematical model used for programming the function generators of all components of the COSY ring, especially the cavity's RF and the dipole-currents.

The beam momentum only depends on the revolution frequency $\nu$ and the closed-orbit length $C$ of the stored beam:

$$
p=m_{p} \beta \gamma c=m_{p} c\left(\left[\frac{c}{C \nu_{\mathrm{RF}}}\right]^{2}-1\right)^{-\frac{1}{2}}
$$

Since COSY operates on the first harmonic the revolution frequency is equal to $\nu_{\mathrm{RF}}$. The RF-frequency was recorded during data taking, and perfect agreement with its nominal value was found. Time stamps were recorded with the data every $2.5 \mathrm{~ms}$ using a high-precision $20 \mathrm{MHz}$ clock (relative accuracy of $10^{-6}$ ). Thus, based on the ramping speed of $1.15 \mathrm{MeV} / \mathrm{c}$ per ms, the momentum for individual events is known better than $\pm 1.5 \mathrm{MeV} / c$. The remaining uncertainty is due to the closed-orbit length $C$ and its possible momentum dependence. Upper limits on its deviation from the ideal orbit length $\left(C_{\mathrm{i}}=183.472 \mathrm{~m}\right)$ were deduced from measurements of the beam positions as a function of time at 29 locations around the ring. With the parameters of the COSY-lattice the maximal change in $C$ compatible with constraints from ion-optics was deduced. The corresponding relative momentum deviation is at most $3 \cdot 10^{-4}$, i.e. less than $1 \mathrm{MeV} / c$ at $3.4 \mathrm{GeV} / c$ [40]. A correction of $C$ with respect to $C_{\mathrm{i}}$ turned out to be negligible.

\subsection{Dead-Time Correction}

The dead time fraction of the data-acquisition system was typically $90 \%$ and its instantaneous value had to be determined accurately. Using fast $(20 \mathrm{MHz})$ scalers we recorded both the number of events read-out $N_{R}$ and the number of all trigger $N_{T}$ (about $10 \mathrm{kHz}$ ) dead-time free. The dead time fraction is then given by $\tau(t)=\left(1-N_{R} / N_{T}\right)$ with statistical uncertainties of less than $0.5 \%$. Systematic errors were checked by taking data samples with data rates varying by orders of magnitude and turned out to be negligible. 


\subsection{Combination of Data Samples and Consistency Checks}

All 17 data samples were analyzed separately, the relative luminosity was fixed using the SEM-monitor and the absolute cross section normalization with respect to [30] as described in Sect.3.9.4 These data samples are distinguished by at least one of the following: the time of the measurement, the $\mathrm{CH}_{2}$-target used, COSY-beam parameters and trigger conditions. This allows to test for systematic variations of the results with these parameters. Let us introduce the shorthand-notations $\sigma_{i}$ and $\delta \sigma_{i}$ for the differential cross section of the i'th data sample and its error. The compatibility of the results $\left\{\sigma_{i}\right\}$ of $n$ data samples, i.e. $i=1, \ldots, n$, was tested by looking at the individual contributions to the total $\chi^{2}$ when minimizing

$$
\chi^{2}=\sum_{i=1}^{n} \sum_{j, k} \frac{\left(\mathcal{N}_{i} \sigma_{i}\left(\theta_{j}, p_{k}\right)-\overline{\sigma_{j, k}}\right)^{2}}{\mathcal{N}_{i}^{2} \delta \sigma^{2}\left(\theta_{j}, p_{k}\right)}
$$

by variation of the normalization factors $\mathcal{N}_{i}$ with $\mathcal{N}_{1}$ fixed to unity. Here, the mean cross section is then given by the weighted mean

$$
\overline{\sigma_{j, k}}=\sum_{i=1}^{n} \frac{\mathcal{N}_{i} \sigma_{i}\left(\theta_{j}, p_{k}\right)}{\mathcal{N}_{i}^{2} \delta \sigma^{2}\left(\theta_{j}, p_{k}\right)} / \sum_{i=1}^{n} \frac{1}{\mathcal{N}_{i}^{2} \delta \sigma^{2}\left(\theta_{j}, p_{k}\right)}
$$

The $\mathcal{N}_{i}$ were treated as free parameters, so that the statistical accuracy $\delta \sigma^{2}\left(\theta_{j}, p_{\text {ref }}\right)$ at the normalization momentum, dominating the absolute normalization error of the individual data samples, do not contribute to the $\chi^{2}$. The fitted values for the $\mathcal{N}_{i}$ turn out to be very close to unity, well within the uncertainty due to $\delta \sigma^{2}\left(p_{\text {ref }}\right)$.

When all data samples were combined the total $\chi^{2}$ per degree of freedom was 1.03. When testing the influence of certain aspects of the analysis on the final results, samples with the same conditions with respect to the aspect under study were combined viz Eq. [23 and renormalized to [30] in order to increase the statistical accuracy of the consistency check. An example is shown Fig. 18 where the dependence on the COSYbeam setup is tested, showing consistent results within the statistical errors. No systematic deviation of the results from different samples could be determined. In addition cuts applied to the data have been varied within reasonable limits to check for possible systematic dependencies of the final result on details of the analysis. As an example, the value of $\alpha_{\text {cut }}$, the maximum allowed value for the kinematic deficit may be increased by $3^{\circ}$ without changing the results significantly, although at the expense of increased statistical error. The change is always much smaller than the estimated systematic error and amounts to less than $1 \%$ for the majority of the data.

Final cross sections are obtained by combining all 17 samples with the help of Eq. 23) and renormalizing them at $p_{\text {ref }}$ to [30]. These data comprise a total of $37 \cdot 10^{6}$ pp elastic scattering events.

\subsection{Errors and Uncertainties}

The calculation of the excitation functions receives error contributions from all factors on the right hand side of Eq. (3). We distinguish five different kinds of errors:
1. statistical errors, due to the number of observed counts from $\mathrm{CH}_{2}$ - and $\mathrm{C}$-targets as in Eq. (8) with a very small contribution from the relative luminosity $\mathcal{L}$ of the carbon-sample. This error ranges from $1 \%$ at small $\theta_{\text {c.m. }}$ and small momenta to $7 \%$ at $\theta_{\text {c.m. }} \approx 90^{\circ}$ and large momenta reflecting the functional dependence of $\mathrm{d} \sigma . / \mathrm{d} \Omega_{\mathrm{c} . \mathrm{m}}$.

2. systematic errors which are treated as uncorrelated between data points adjacent in scattering angle or momentum. Here, artefacts from the reconstruction of the scattering angles contribute. The angular resolution of the EDDA detector is not entirely homogeneous, but varies slightly with $Q_{12}$ (cf. Eq. (5)). When the data are binned in $\theta_{\text {c.m. }}$ an uncertainty of $2.4 \%$, usually anti-correlated for neighboring bins, arises. Smaller contributions to this uncertainty stems from the cut on the azimuthal angle $\phi(0.6 \%)$ and the loss of events due to the cut on $P_{\text {el. }}(0.5 \%)$.

3. systematic errors associated to undetected elastic scattering events and misidentified inelastic events. This uncertainty will have a smooth dependence on scattering angle and beam momentum. The detection efficiency, obtained by MC methods, contributes with $1.5 \%$ while possible contamination with inelastic events account for an uncertainty increasing with beam momentum and scattering angle from less than $1 \%$ to $5.5 \%$. These contributions are always smaller than the first two errors combined.

4. a relative normalization error, common to all data points at the same momentum of $2.5 \%$, derived from the maximum discrepancy observed in the relative luminosity determination of the SEM and PIN monitors. The statistical error of the SEM signal as well the uncertainty in determination of the DAQ dead-time are negligible in comparison. Uncertainties in the correction $\bar{\rho} / \rho_{0}$, due to uncertainties in $\lambda_{1,2}$ of Eq. (20) and the applied dose, enter only as the ratio to its value at $p_{\text {ref }}$, so that its contribution is less than $0.3 \%$ for all beam momenta.

5. an absolute normalization error common to all data of less than $1.5 \%$, comprising the uncertainty of $\sigma_{\text {ref }}$ of Eq. (21) and the statistical errors of our data at $p_{\text {ref. }}$.

In all figures presenting the data of this work, only the first two errors are shown, i.e. the statistical error and an additional $2.5 \%$ systematic error. All errors are listed in [41].

\section{Experimental Results}

The experimental results of the present elastic $p p$ scattering experiment are the unpolarized differential cross sections $\mathrm{d} \sigma / \mathrm{d} \Omega_{\mathrm{c} . \mathrm{m}}$. as a function of the c.m.-scattering angle $\theta_{\text {c.m. }}$ and the laboratory momentum $p$ of the proton beam. Each differential cross section refers to bins $\Delta \theta_{\text {c.m. }}=2^{\circ}$ and $\Delta p=25 \mathrm{MeV} / \mathrm{c}$, which were chosen to have reasonable statistical precision for each data point. We covered a beam momentum range of $0.7-$ $3.4 \mathrm{GeV} / \mathrm{c}$ corresponding to laboratory kinetic energies $0.23-$ $2.59 \mathrm{GeV}$ and total c.m. energies $\sqrt{s}=2.0-2.9 \mathrm{GeV}$. Since in pp elastic scattering the final state particles are indistinguishable and cross sections are symmetric with respect to $\theta_{\text {c.m. }}=$ $90^{\circ}$, data are only given for the c.m. angular range $34^{\circ}-90^{\circ}$. All 2888 data points are available online through the worldwide web [41]. They may be viewed as 28 excitation functions 


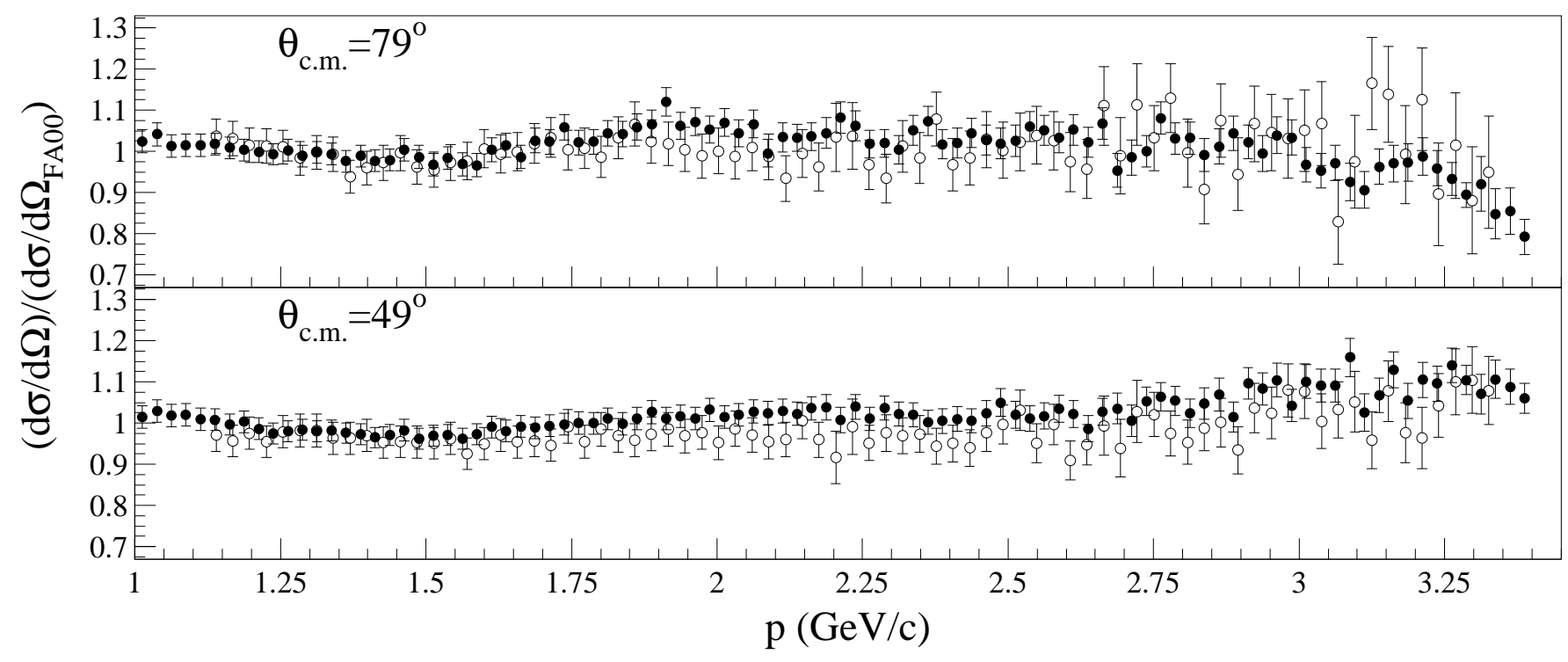

Fig. 22. Comparison of the differential cross section of [14] (open symbols) with this work (closed symbols) at two angles. To remove the main energy dependence all cross sections have been divided by the PSA-prediction of [8] (solution FA00).

(cf. Fig. 23 at c.m. scattering angles between $34^{\circ}$ and $90^{\circ}$ or 108 angular distributions (cf. Fig. 25) at 108 beam momenta between 0.7 and $3.4 \mathrm{GeV} / \mathrm{c}$. Note, that due to kinematics the angular acceptance is reduced at very small and very high beam momenta.

As compared to our previously published results [14], based on a subset of the data, the momentum range is increased from $1.1-3.3 \mathrm{GeV} / \mathrm{c}$ to $0.7-3.4 \mathrm{GeV} / \mathrm{c}$ and the statistical precision increased by up to a factor of 3 . In addition, in the analysis of Ref. [14] the variation of the hydrogen density along the target due to radiation damage was not corrected for, this led to a systematic underestimation of the cross section by about $5 \%$ above $2 \mathrm{GeV} / \mathrm{c}$, as displayed in Fig. 22 Note, that the results from [14] are superseded by this work and should not be used anymore.

\subsection{Comparison to Other Data}

In Fig. 24 the available database on pp elastic scattering (cf. [7, 8, 42, 43, 44] and references therein) is plotted for the same angles as for the data of the present work in Fig. 23 The benefit of a consistent normalization, made possible by measuring during acceleration in an internal experiment, as well as the improved statistical accuracy is evident. Previous data scatter considerably around available phase-shift solutions (discussed in detail in Sect.5.2 partly due to larger statistical errors, but mainly due to differences in the absolute cross section normalization of the various experiments. Most notably the data of Jenkins et al. [45] from the ZGS are lower by about $20 \%$ ( $\star$, $;$ in Fig. 24 and clearly disagree with our data as well as those of [46, 47]. In Fig. 26 angular distributions are compared at four momenta to data from other experiments. The shapes of the differential cross section are consistent within quoted uncertainties, however, again absolute normalizations are at variance. To show the size of this scale difference we have renor- malized our data using the procedure described in Sect. 3.9.4 to match the normalization of other experiments which spanned a larger range of beam momenta. The result is displayed in Fig. 27 and shows that the data of Kammerud et al. [46] are consistently higher by $10 \%$, the data of Williams et al. [47] agree, within the sizeable uncertainties, and the data from Albrow et al. [48] are partly compatible with our normalization. On the average these data tend to be larger at momenta above about $1.5 \mathrm{GeV} / \mathrm{c}$, with the exception of the data of Jenkins et al. [45] discussed earlier. The excitation function of Garcon et al. [49] at $\theta_{\text {c.m. }} \approx 90^{\circ}$, measured by a similar technique, shows the opposite trend (Fig. 28) and cross sections are smaller by up to 2 standard deviations.

Note, that all 2888 cross section data obtained in this experiment share a common absolute normalization factor. In case data becomes available in the future, with a superior method to obtain an absolute normalization, our data could be renormalized by a common factor to be determined along the same lines as described in Sect. 3.9.4 The relative normalization uncertainty of $2.5 \%$ would not be affected.

\section{Discussion}

Theoretical models of the nucleon-nucleon interaction have been very successful in describing elastic scattering data at energies where cross sections of inelastic reactions are still small. In this domain $\left(T_{p}\right.$ below about $\left.0.5 \mathrm{GeV}\right)$ high-precision potentials, purely phenomenological or based on the meson-exchange picture, are available (for a review see Ref. [62]). More recently effective field-theory has entered the stage and produced results of comparable quality, cf. Refs. [63, 64, 65] and references therein. However, in the energy region between $0.5 \mathrm{GeV}$ and a few $\mathrm{GeV}$ little progress has been made in recent years. In the late $80 \mathrm{~s}$, the meson exchange models were extended to higher 


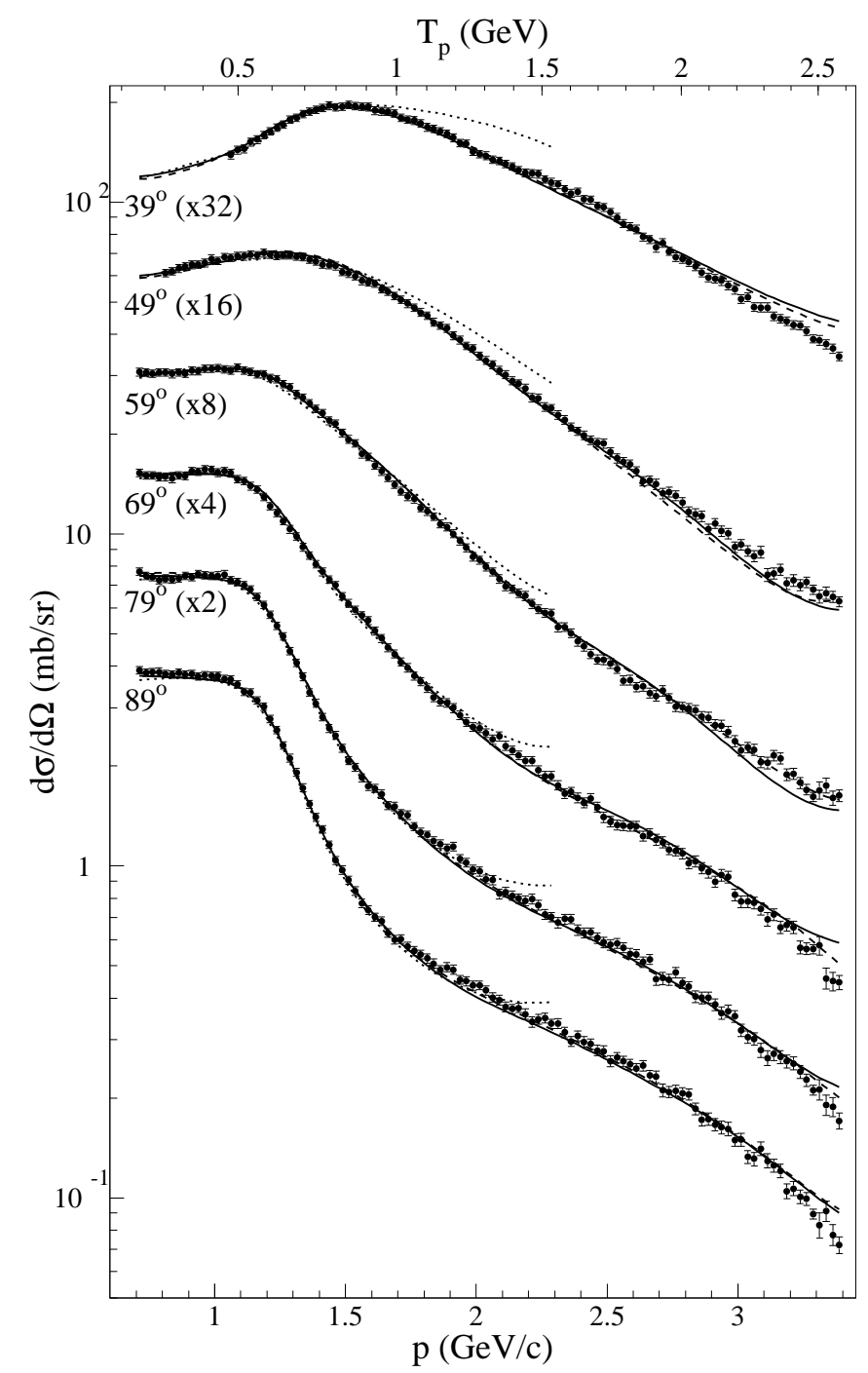

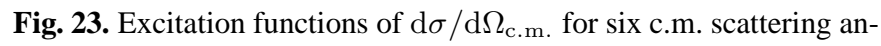
gles in comparison to SAID solutions SM94 (dotted), SM97 (dashed) and FA00 (solid line).

energies by including inelastic reactions by coupling to intermediate $N \Delta$ and $\Delta \Delta$ channels [66, 67]. A qualitative description of the $N N$-data below $1 \mathrm{GeV}$ has been achieved. However, at higher energies these models fail badly and differential cross sections at large scattering angles are strongly overestimated [68]. This may not be surprising since inelasticities at these energies will no longer be driven predominantly by the $\Delta(1232)$ resonance. Work aiming to improve these models are currently under way [69].

On the high-energy side optical potential models and Reggephenomenology has been used to describe the gross features of the unpolarized differential cross section [45, 46]. Dimensional scaling [70] predicts cross sections to follow a $s^{-10}$ behavior at large momentum transfer. Our data are too low in momentum transfer for these models to be strictly applicable. Nonetheless, in Fig. 29 we compare our differential cross sections, plotted as $\frac{\mathrm{d} \sigma}{\mathrm{dt}} \mathrm{vs}-t$, to the prediction of [70] (solid line, note that for $\theta_{\text {c.m. }}=90^{\circ}$ we have $s=4 m_{p}^{2}-2 t$ ), showing that we may

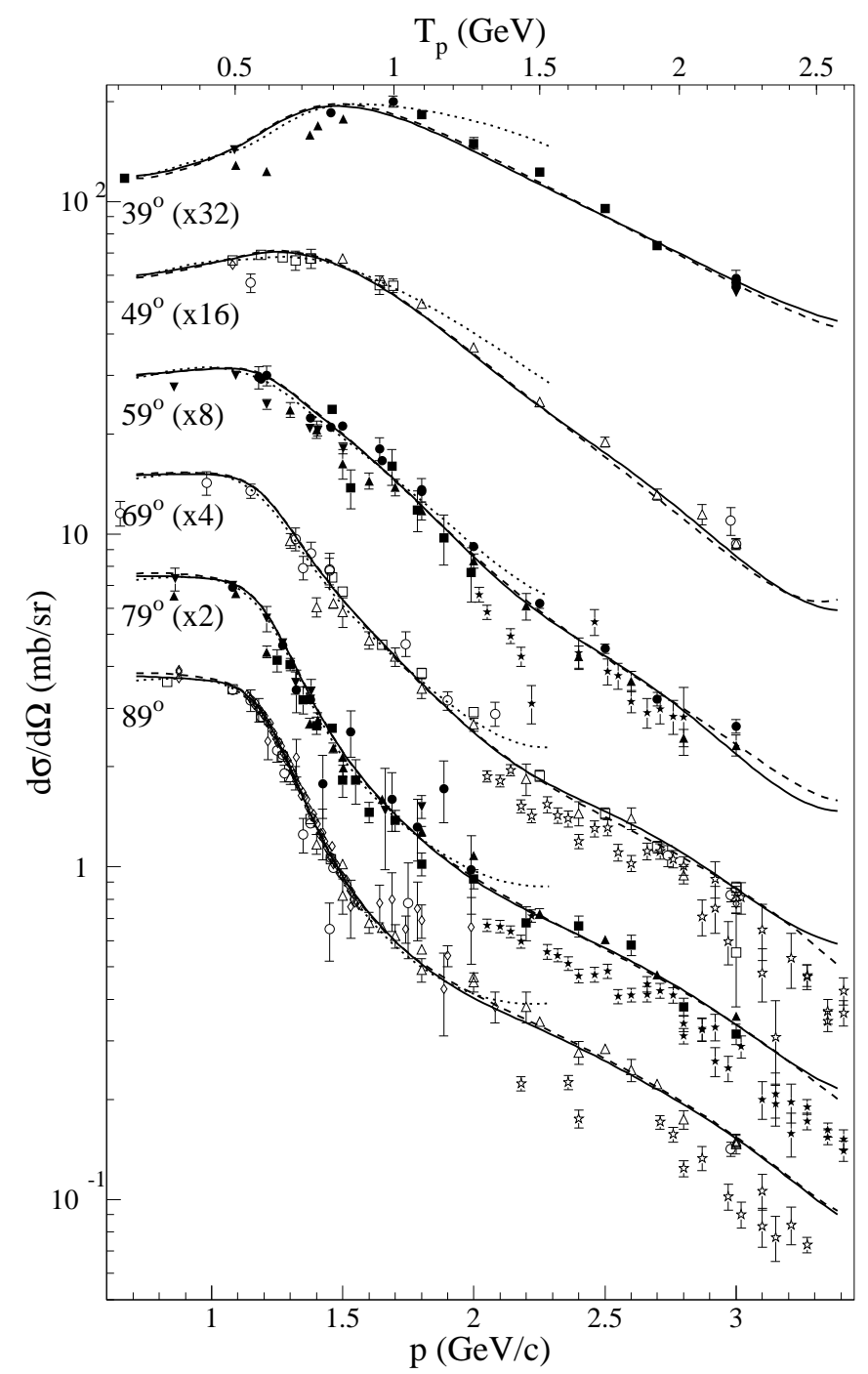

Fig. 24. Collection of published data (From the database of [8] without EDDA data of Albers et al. [14]) plotted as excitation functions at six angles $\left( \pm 1^{\circ}\right)$ in comparison to phase shift predictions as in Fig. 23

be barely touching the region of dimensional scaling, in agreement with [45].

The immediate benefit of the high-precision data obtained in this experiment is two-fold: first, its consistent normalization allow for stringent tests of energy-dependent structures, as they could arise from coupling to an intermediate resonant state, and secondly it will further help to consolidate phase-shift parameters for the isotriplet elastic $\mathrm{NN}$-channel.

\subsection{Upper Limits on Resonant Contributions}

All excitation functions show a smooth and rather structureless dependence on beam momentum. No sharp energy-dependent structure is observed which could be taken as evidence for a narrow resonance. Therefore, upper limits for the elasticities $\Gamma_{e l} / \Gamma$ of hypothetical narrow resonances are deduced from the smooth excitation functions. To this end a Breit-Wigner resonance term is introduced into the scattering matrix element of 


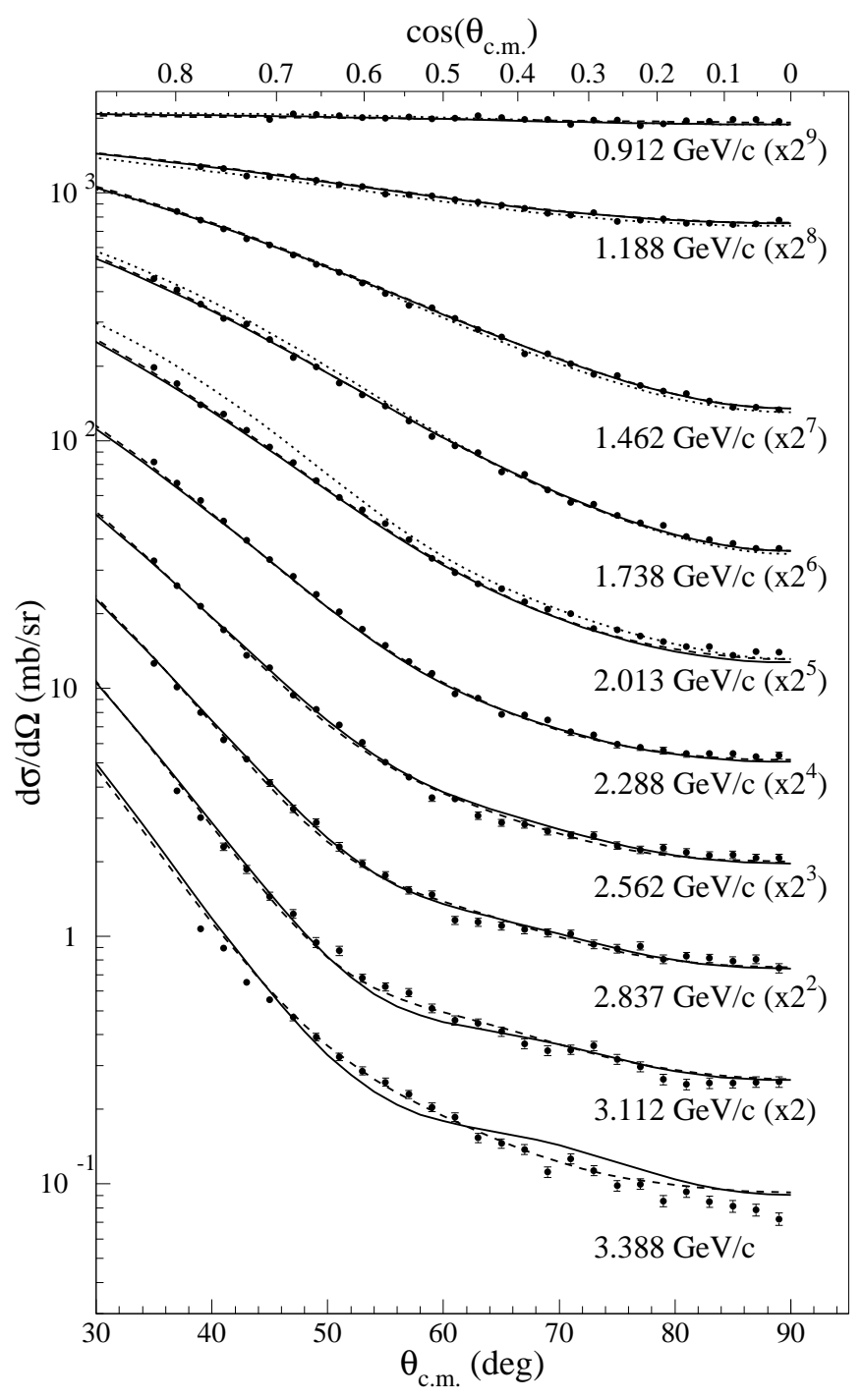

Fig. 25. Angular distributions of $\mathrm{d} \sigma / \mathrm{d} \Omega_{\mathrm{c} . \mathrm{m} \text {. }}(\bullet$ : data of this work) for ten beam momenta $p$ in in comparison to SAID solutions (cf. Fig.23.

the partial wave which is assumed to exhibit resonance behavior. The interference between the Breit-Wigner resonance term and the non-resonant amplitudes determines the size of a resonance excursion in the excitation functions. The non-resonant amplitudes represent the null-hypothesis. In order to establish the null-hypothesis the EDDA data are fitted by a special energy dependent phase shift analysis along Ref.[8].

In the test calculations the hypothetical resonance energy $E_{R}$, total width $\Gamma$ and resonance phase $\phi_{R}$ are varied systematically in the range $E_{R}=2.2-2.8 \mathrm{GeV}, \Gamma=10-100 \mathrm{MeV}$ and $\phi_{R}=0^{\circ}-360^{\circ}$. The hypothesis of the existence of a resonance in a partial wave is tested by gradually increasing the partial elastic width $\Gamma_{e l}$ until the resonance is excluded within $99 \%$ confidence level by a $\chi^{2}$-test based on the the differential cross sections and analyzing power data [15]. For the unknown phase $\phi_{R}$ the value giving the largest, and thus most conservative limit on $\Gamma_{e l} / \Gamma$ is chosen. Typical upper limits of $\Gamma_{e l} / \Gamma$ for the five lowest uncoupled partial waves are 0.08 for ${ }^{1} \mathrm{~S}_{0}$,

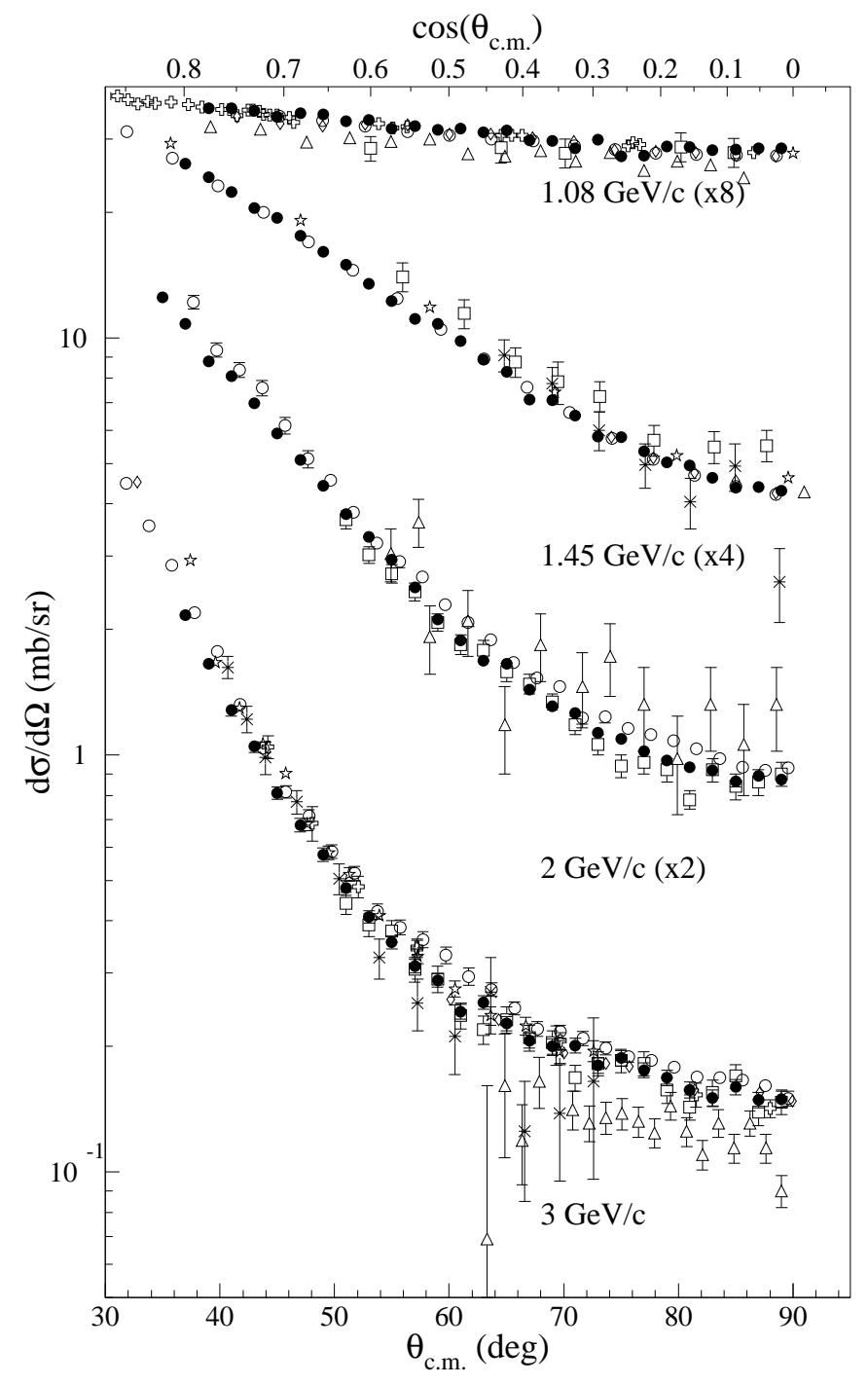

Fig. 26. Angular distributions at four beam momenta. Data of this work $(\bullet)$ are compared to published data, where the symbols $\bigcirc, \square$, $\triangle, \diamond, 々$, $九$, and $*$ correspond to data from $[30,48,50,51,52,53]$ at $1.08 \mathrm{GeV} / \mathrm{c},[30,48,49,51,54,55,56]$ at $1.45 \mathrm{GeV} / \mathrm{c},[46,47,57]$ at $2 \mathrm{GeV} / \mathrm{c}$ and $[45,46,47,58,59,60,61]$ at $3 \mathrm{GeV} / \mathrm{c}$.

0.04 for ${ }^{1} \mathrm{D}_{2}, 0.10$ for ${ }^{3} \mathrm{P}_{0}, 0.03$ for ${ }^{3} \mathrm{P}_{1}$ and 0.05 for ${ }^{3} \mathrm{~F}_{3}$. For instance the ${ }^{1} \mathrm{~S}_{0}$ dibaryon resonance predicted by Lomon et al. [10] at $E_{R}=2.7 \mathrm{GeV}$ with $\Gamma_{e l} / \Gamma=0.1$ and $\Gamma=50 \mathrm{MeV}$ can safely be excluded. The method is described in detail in a forthcoming paper [26].

It should be noted in this context that two broad resonant structures are well known at lower energies, the ${ }^{1} \mathrm{D}_{2}$ resonance at $E_{R} \approx 2.15 \mathrm{GeV}$ and the ${ }^{3} \mathrm{~F}_{3}$ resonance at $E_{R} \approx 2.17 \mathrm{GeV}$ with widths of around $120 \mathrm{MeV}$. They appear as counterclockwise circles in the Argand diagrams of the phase shift analysis [42] of the world data set. Especially the excitation functions of the spin-dependent total cross sections $\Delta \sigma_{L}$ and $\Delta \sigma_{T}$ measured at ZGS [71, 72] show marked energy-dependent structures. These ${ }^{1} \mathrm{D}_{2}$ and ${ }^{3} \mathrm{~F}_{3}$ resonances in the $p p$-system can be interpreted conventionally by intermediate ${ }^{5} \mathrm{~S}_{2}$ and ${ }^{5} \mathrm{P}_{3} N \Delta$ 


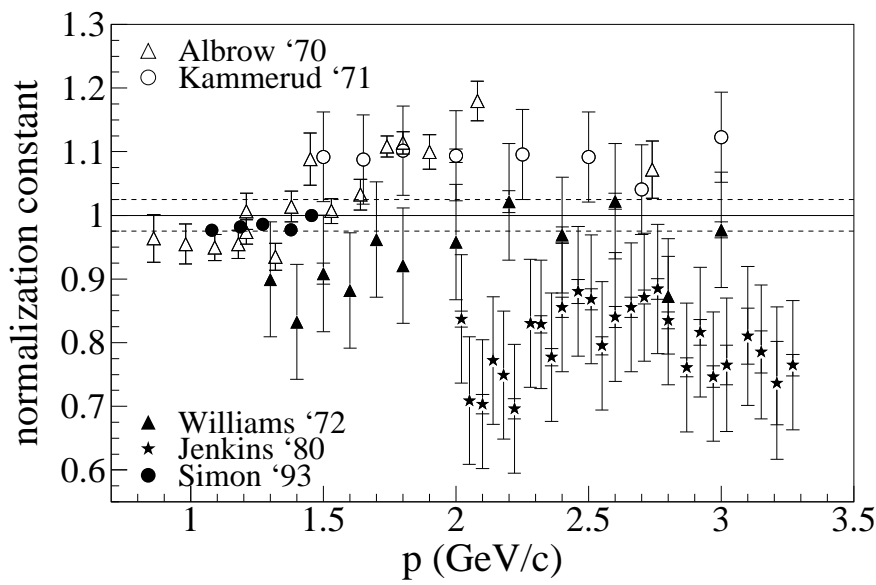

Fig. 27. Comparison of the absolute normalization constant relative to [30] at $1.455 \mathrm{GeV} / \mathrm{c}$ for angular distributions measured at CERN (Albrow et. al [48]), the Argonne ZGS (Kammerud et. al [46]), Rutherford (Williams et al. [47]), Brookhaven (Jenkins et. al [45]), and LAMPF (Simon et al. [30]). The smaller error bars show statistical uncertainties only and the larger error bars contain the normalization uncertainty added in quadrature. The dashed horizontal line shows the relative normalization error of this experiment.

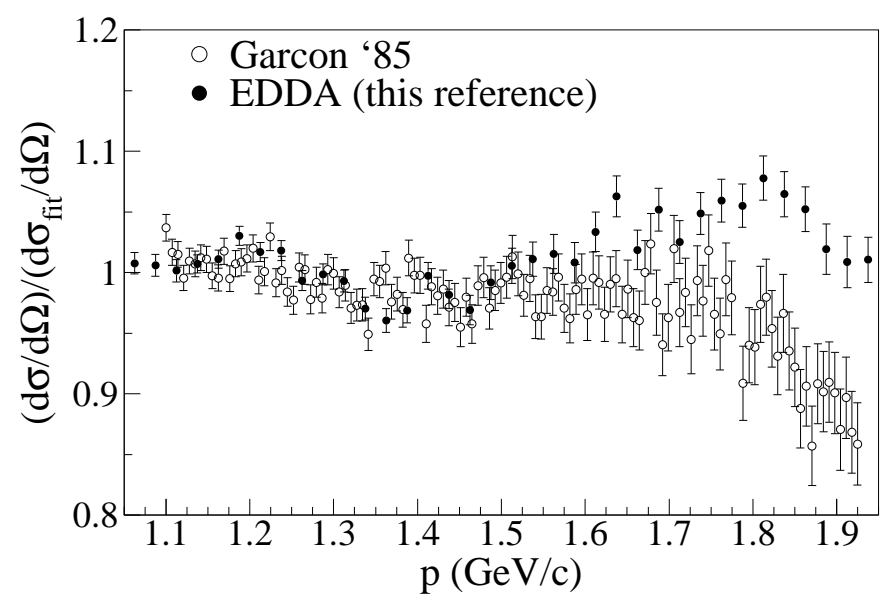

Fig. 28. Comparison to the excitation function of [49] at $\theta_{\text {c.m. }} \approx 90^{\circ}$. The EDDA date have been interpolated to the exact angle as in [49] and all cross sections have been divided by $\mathrm{d} \sigma_{\text {fit }} / \mathrm{d} \Omega_{\text {c.m. }}$, a smooth parameterization of the data.

states $[66,73,74,75,76]$. These broad resonances show up as a rather rapid change in the shape of the angular distribution around $1.25 \mathrm{GeV} / \mathrm{c}(\sqrt{s}=2.15 \mathrm{GeV})$ where the cross section exhibit a steep ascend (descend) at forward (backward) angles.

\subsection{Impact on phase shift analysis}

The excitation functions of the differential cross sections are measured over a wide momentum range from 0.7 to $3.4 \mathrm{GeV} / \mathrm{c}$. They have a great impact on the energy-dependent phase shift analysis since they represent a precise and consistent data set of 2888 data points. Another important fact is that the unpolar-

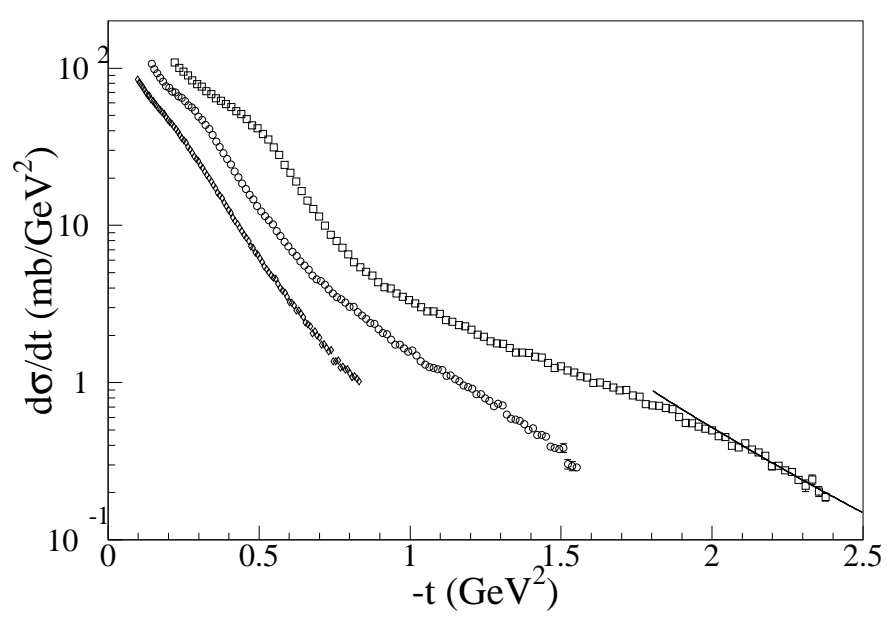

Fig. 29. Differential cross section $\frac{\mathrm{d} \sigma}{\mathrm{dt}}$ at 3 selected c.m. scattering angles: $89^{\circ}(\square), 69^{\circ}(\bigcirc)$, and $49^{\circ}(\diamond)$.

ized differential cross sections fixes the global scale of all amplitudes and thus enters repeatedly into the phase shift analysis through all spin dependent cross sections, which are products of spin-observables with the unpolarized cross section.

The dotted curves in Fig. 23 are phase shift solutions SM94 of Arndt et al. [6] from summer 1994, i.e. before the first EDDA data were available. The maximum kinetic energy of this phase shift solution was $1.6 \mathrm{GeV}$ corresponding to a beam momentum of $2.36 \mathrm{GeV} / \mathrm{c}$. After the publication of the first EDDA data [14] the VPI group extended their energy dependent phase shift analysis from $1.6 \mathrm{GeV}(2.36 \mathrm{GeV} / \mathrm{c})$ up to $2.5 \mathrm{GeV}(3.3 \mathrm{GeV} / \mathrm{c})$, with the solution SM97. Meanwhile an energy dependent phase shift solution FA00 is available up to $3.0 \mathrm{GeV}(3.82 \mathrm{GeV} / \mathrm{c})$ laboratory kinetic energy (beam momentum) [8]. The phase shift solutions SM97 and FA00 are shown as dashed and solid curves in Figs. 23 and 25, respectively. Phase-shift predictions show an apparent oscillation both in angle and beam momentum about the data of this work. This may be an artefact of the current parameterization of the energy-dependence of phaseshifts. Therefore, including the data of the present work will not only slighly modify the phase-shifts to reflect the differences to our previous results ([14] and Fig. 22] but may also allow to improve the ansatz for the variation of the phase shift parameters with energy. In this process a better description of the angular distribution at the higher momenta should be attempted.

\section{Summary}

In a dedicated experiment, protons accelerated in a synchrotron have been scattered of internal $\mathrm{CH}_{2}$-fiber targets during accelaration at beam momenta between 0.7 and $3.4 \mathrm{GeV} / \mathrm{c}\left(T_{p}=\right.$ $0.23 \ldots 2.59 \mathrm{GeV})$. Elastic scattering events have been identified by the EDDA-detector over a wide angular range $\left(\theta_{\text {c.m. }}=\right.$ $\left.34^{\circ} \ldots 90^{\circ}\right)$. This experimental technique allows a precise monitoring of the relative change in luminosity with beam momentum which leads to a consistent normalization of data taken at different momenta and is therefore ideally suited to measure 
excitation functions with high precision. The absolute cross section scale was fixed at the reference momentum $1.455 \mathrm{GeV} / \mathrm{c}$ to high-precision data from LAMPF [30]. The average combined statistical and systematic error is $3.8 \%$, it ranges from $3 \%$ for small momenta and scattering angles to $10 \%$ at high momenta and $\theta_{\text {c.m. }}=90^{\circ}$. The total absolute normalization uncertainty of the complete data set is below $1.5 \%$ with an additional relative normalization uncertainty of $2.5 \%$ common to all data points at the same beam momentum.

The wealth of new data obtained in this experiments replaces the data reported earlier [14] and utilized improved analysis methods to yield higher statistical and systematic precision, most notably at higher energies. These data will further improve extracted phase-shift parameters and provide an important normalization standard for measurements of $\mathrm{NN}$ reactions. The data are available via the world-wide web [41].

The excitation-functions obtained with steps of $25 \mathrm{MeV} / \mathrm{c}$ in beam-momentum (or $\Delta \sqrt{s} \approx 8.5 \mathrm{MeV}$ ) have been applied in tests for contributions of narrow resonances, like dibaryons, coupling to the elastic channel. No evidence for any narrow (10-100 MeV) structures have been found in the invariant mass range $2.0 \ldots 2.8 \mathrm{GeV}$ covered by the experiment, ruling out a strong coupling of such resonances - if they exist - to the elastic channel.

We thank the operating team of COSY for excellent beam support. This work was supported by the German BMBF, contracts 06BN664I(6) and $06 \mathrm{HH} 852$, and by the Forschungszentrum Jülich (Aufträge 411 26803 and 41126903).

\section{References}

1. C. Lechanoine-LeLuc and F. Lehar, Rev. Mod. Phys. 65 (1993).

2. W. Haeberli et al., Phys. Rev. C55, 597 (1997).

3. B. von Przewoski et al., Phys. Rev. C58, 1897 (1998).

4. F. Rathmann et al., Phys. Rev. C58, 658 (1998).

5. J. Ball et al., CTU Reports 4, 3 (2000).

6. R. A. Arndt, I. I. Strakovskii, and R. L. Workman, Phys. Rev. C50, 2731 (1994).

7. R. A. Arndt, C. H. Oh, I. I. Strakovsky, R. L. Workman, and F. Dohrmann, Phys. Rev. C56, 3005 (1997), nuclth/9706003.

8. R. A. Arndt, I. I. Strakovsky, and R. L. Workman, Phys. Rev. C 62, 34005 (2000).

9. J. Ball et al., Phys. Lett. B 320, 206 (1994).

10. P. Gonzalez, P. LaFrance, and E. L. Lomon, Phys. Rev. D35, 2142 (1987).

11. P. J. G. Mulders, A. T. M. Aerts, and J. J. de Swart, Phys. Rev. Lett. 40, 1543 (1978).

12. P. J. Mulders, A. T. Aerts, and J. J. D. Swart, Phys. Rev. D21, 2653 (1980).

13. P. LaFrance and E. L. Lomon, Phys. Rev. D34, 1341 (1986).

14. D. Albers et al. (EDDA), Phys. Rev. Lett. 78, 1652 (1997).

15. M. Altmeier et al. (EDDA), Phys. Rev. Lett. 85, 1819 (2000).

16. F. Bauer et al. (EDDA), Phys. Rev. Lett. 90, 142301 (2003).
17. R. Maier, Nucl. Instr. and Meth. A390, 1 (1997).

18. K. Ackerstaff et al. (EDDA), Nucl. Instrum. Meth. A335, 113 (1993).

19. J. Bisplinghoff et al. (EDDA), Nucl. Instr. and Meth. A329, 151 (1993).

20. D. Albers et al. (EDDA), Nucl. Instr. and Meth. A 371, 388 (1996).

21. H. Scheid, Dissertation, Institut für Strahlen- und Kernphysik, Universität Bonn (1994), see: http://www.iskp.uni-bonn.de/edda/dipldiss.html

22. F. Hinterberger and D. Prasuhn, Nucl. Instr. and Meth. A279, 413 (1989).

23. J. Lindlein, Diplomarbeit, I. Exp. Phys., Universität Hamburg (1996), see, http://kaa.desy.de/edda/papers/Diplomalist.inhalt.html

24. J. P. Coffin, Experimental Techniques in Nuclear Physics (de Gruyter, Berlin, 1997), p. 309.

25. K. Ackerstaff et al. (EDDA), Nucl. Instrum. Meth. A491, 492 (2002).

26. H. Rohdjeß et al. (EDDA), nucl-ex/0403043.

27. H.-H. Bothe, Fuzzy Logic - Einführung in Theorie und Anwendungen (Springer, Heidelberg, 1995).

28. F. Höppner, F. Klawonn, R. Kruse, and T. Runkler, Fuzzy Cluster Analysis (Wiley, New York, 1999).

29. M. Busch, Dissertation, ISKP, Universität Bonn (2001) see: http://www.iskp.uni-bonn.de/edda/dipldiss.html

30. A. J. Simon et al., Phys. Rev. C 48, 662 (1993).

31. E. J. Sternglass, Phys. Rev. 108, 1 (1957).

32. Particle-Data-Group, C. Caso et al. (Particle Data Group), Eur. Phys. J. C3, 1 (1998), see also http://pdg.lbl.gov

33. M. N. Rosenbluth, Phys. Rev. 79, 615 (1950).

34. G. Kallen, Elementary Particle Physics (Addison-Wesley, 1964).

35. T. Hüskes, Diplomarbeit, ISKP, Universität Bonn (1997), see: http://www.iskp.uni-bonn.de/edda/dipldiss.html

36. W. R. Nelson, H. Hirayama, and D. W. O. Rogers, The EGS4 Code System (1985), SLAC-Report 265, URL: 'http://www.slac.stanford.edu/egs/'.

37. R. Groß-Hardt, Dissertation, Institut für Strahlen- und Kernphysik, Universität Bonn (2001).

38. H. Rohdjeß et al. (EDDA), nucl-ex/0403044.

39. F. Hinterberger and D. Prasuhn, Nucl. Instr. and Meth. A321, 453 (1992).

40. H. P. Engelhardt, Dissertation, Institut für Strahlen- und Kernphysik, Universität Bonn (1998), see: http://www.iskp.uni-bonn.de/edda/dipldiss.html

41. Data can be accessed via http://kaa.desy.de and http://www.iskp.uni-bonn.de/gruppen/edda

42. R. A. Arndt, L. D. Roper, R. L. Workman, and M. W. McNaughton, Phys. Rev. D45, 3995 (1992).

43. R. A. Arndt, J. M. Ford, and L. D. Roper, Phys. Rev. D32, 1085 (1985).

44. R. A. Arndt, W. J. Briscoe, R. L. Workman, and I. I. Strakovsky, SAID: Scattering Analysis Interactive Dialin (2004), http://gwdac.phys.gwu.edu/analysis/nn_analysis.

45. K. A. Jenkins et al., Phys. Rev. D 21, 2445 (1980).

46. R. C. Kammerud et al., Phys. Rev. D 4, 1309 (1971).

47. D. T. Williams et al., Nuovo Cimento 8A, 447 (1972).

48. M. G. Albrow et al., Nucl. Phys. B 23, 445 (1970). 
49. M. Garcogn et al., Nucl. Phys. A 445, 669 (1985).

50. K. Abe et al., Phys. Rev. D12, 1 (1975).

51. A. J. Simon et al., Phys. Rev. C 53, 30 (1996).

52. G. W. Hoffmann et al., Phys. Rev. C37, 397 (1988).

53. D. Ottewell et al., Nucl. Phys. A412, 189 (1984).

54. A. v. Dobrovolsky et al., Nucl. Phys. B214, 1 (1983).

55. M. 1. Barlett et al., Phys. Rev. C27, 682 (1983).

56. B. A. Ryan, A. Kanofsky, T. J. Devlin, R. E. Mischke, and P. F. Shepard, Phys. Rev. D3, 1 (1971).

57. F. Shimizu et al., Nucl. Phys. A, 445 (1982).

58. A. R. Clyde, Lawrence Livermore Radiation Laboratory Report No. UCRL-16275 (1966), unpublished.

59. C. M. Ankenbrandt et al., Phys. Rev. 170, 1223 (1968).

60. D. R. Rust et al., Phys. Rev. Lett. 24, 1361 (1970).

61. I. Ambats et al., Phys. Rev. D 9, 1179 (1974).

62. R. Machleidt, in Adv. Nucl. Phys., edited by J. W. Negele and E. Vogt (Plenum Press, New York, 1989), vol. 19, chap. 2, pp. 189-376.

63. U. van Kolck, Prog. Part. Nucl. Phys. 43, 337 (1999).

64. E. Epelbaum, W. Glöckle, and U.-G. Meissner, Nucl. Phys. A671, 295 (2000), nucl-th/9910064.

65. D. R. Entem and R. Machleidt, Phys. Rev. C68, 041001 (2003), nucl-th/0304018.

66. C. Elster, K. Holinde, D. Schütte, and R. Machleidt, Phys. Rev. C38, 1828 (1988).

67. C. Elster, W. Ferchländer, K. Holinde, D. Schütte, and R. Machleidt, Phys. Rev. C37, 1647 (1988).

68. K. O. Eyser, R. Machleidt, and W. Scobel (2003), nuclth/0311002.

69. C. Elster, Ohio University, Athens, Ohio (2003), private communication.

70. S. J. Brodsky and G. R. Farrar, Phys. Rev. Lett. 31, 1153 (1973).

71. I. P. Auer et al., Phys. Rev. Lett. 41, 354 (1978).

72. E. K. Biegert et al., Phys. Lett. 73B, 235 (1978).

73. D. V. Bugg, J. Phys. G 10, 717 (1984).

74. P. Gonzalez and E. L. Lomon, Phys. Rev. D34, 1351 (1986).

75. P. LaFrance, E. L. Lomon, and M. Aw (1993), nuclth/9306026.

76. T. Ueda, Phys. Lett. B119, 281 (1982). 